Article

\title{
A Multi-Objective Optimal Power Flow Control of Electrical Transmission Networks Using Intelligent Meta-Heuristic Optimization Techniques
}

\author{
Hatem Diab ${ }^{1}$, Mahmoud Abdelsalam ${ }^{1, *}$ and Alaa Abdelbary ${ }^{2}$ \\ 1 Electrical Energy Department, Arab Academy for Science and Technology and Maritime Transport, \\ Giza 12212, Egypt; hatem.diab@aast.edu \\ 2 Applied Sciences Department, Arab Academy for Science and Technology and Maritime Transport, \\ Alexandria 2033, Egypt; aaelbary@aast.edu \\ * Correspondence: mahmoud.elwadie@aast.edu
}

check for updates

Citation: Diab, H.; Abdelsalam, M.; Abdelbary, A. A Multi-Objective Optimal Power Flow Control of Electrical Transmission Networks Using Intelligent Meta-Heuristic Optimization Techniques. Sustainability 2021, 13, 4979. https:// doi.org/10.3390/su13094979

Academic Editors:

Maryam Bahramipanah and

Zagros Shahooei

Received: 12 January 2021

Accepted: 24 February 2021

Published: 29 April 2021

Publisher's Note: MDPI stays neutral with regard to jurisdictional claims in published maps and institutional affiliations.

Copyright: (c) 2021 by the authors. Licensee MDPI, Basel, Switzerland. This article is an open access article distributed under the terms and conditions of the Creative Commons Attribution (CC BY) license (https:/ / creativecommons.org/licenses/by/ $4.0 /)$.

\begin{abstract}
Optimal power flow (OPF) is considered one of the most critical challenges that can substantially impact the sustainable performance of power systems. Solving the OPF problem reduces three essential items: operation costs, transmission losses, and voltage drops. An intelligent controller is needed to adjust the power system's control parameters to solve this problem optimally. However, many constraints must be considered that make the design process of the OPF algorithm exceedingly tricky due to the increased number of limitations and control variables. This paper proposes a multi-objective intelligent control technique based on three different meta-heuristic optimization algorithms: multi-verse optimization (MVO), grasshopper optimization (GOA), and Harris hawks optimization $(\mathrm{HHO})$ to solve the OPF problem. The proposed control techniques were validated by applying them to the IEEE-30 bus system under different operating conditions through MATLAB simulations. The proposed techniques were then compared with the particle swarm optimization (PSO) algorithm, which is very popular in the literature studying how to solving the OPF problem. The obtained results show that the proposed methods are more effective in solving the OPF problem when compared to the commonly used PSO algorithm. The proposed HHO, in particular, shows that it can form a reliable candidate in solving power systems' optimization problems.
\end{abstract}

Keywords: optimal power flow; transmission networks; metaheuristic optimization

\section{Introduction}

Electric power networks are very sophisticated and complex systems. This complexity is simply due to the fact that electric power systems consist of three sub-systems, each containing different elements. The first sub-system is the generating stations which mainly consist of generators and their additional items. The second sub-system is the transmission networks which contain transformer substations, transmission lines, and reactive power compensation units. The third sub-system is the distribution networks which are responsible for delivering electric power to the consumers. All these elements have to be operated in a reliable as well as economical manner. Each element of the power system acts as a control variable, as shown in Table 1.

Although all control variables mentioned in Table 1 can be set individually, they influence the power flows of the network. It is essential to mention the power balance between the generation and load consumption when using different setting combinations for the control variables of the power settings. However, the solution of the power flow equation is not enough for the proper operation of the power system as few setting combinations of the power system can achieve the economical and reliable operation of the power system. 
Table 1. Control variable of the electric power system.

\begin{tabular}{clll}
\hline Sub-System & \multicolumn{1}{c}{ Element } & \multicolumn{1}{c}{ Control Variables } \\
\hline \multirow{2}{*}{ Generation } & Generators & $\bullet$ & Active power outputs \\
& & $\bullet$ & Output voltages \\
Transmission & Transformer substations & $\bullet$ & Tap changers \\
& shunt var compensators & $\bullet$ & Reactive power output \\
Distribution & Distribution substation & $\bullet$ & Load bus voltages \\
& & $\bullet$ & Load Reactive power \\
\hline
\end{tabular}

The optimal power flow (OPF) is the process responsible for selecting the setting combination of control variables of the power system to achieve optimal operation. OPF is considered a development of the optimal dispatch concept [1,2]. The objective function of the OPF aims to reduce either the operation cost or system losses and voltage drops through the adjustments of the control variables of the power system. Sometimes, the goal of the objective function is to reduce the three parameters at the same time. This means that the OPF objective function is a non-linear function with many constraints, which makes it not easy to solve. Due to this fact, traditional methods like Newton methods may never find optimum global variables. Additionally, in some cases where the objective function is not available in the algebraic form, the traditional methods fail in solving this type of function [3-9]. Based on this fact, metaheuristic optimization algorithms are highly recommended for solving the OPF problem since they can have the high possibility of finding the globally optimum values for objective functions that have no pre-defined algebraic form.

One of the famous metaheuristic optimization techniques that have been used in the literature in solving the OPF problem is particle swarm optimization (PSO). The PSO, in its basic form, faces some challenges in finding the globally optimum values for the OPF as the initial random allocation of the particles has a significant impact on the success rate of the algorithm [10-13]. A modified PSO algorithm is proposed in [10] to solve this problem by updating the position of the particles from other individuals.

Many researchers have also proposed genetic algorithms (GAs) for solving the OPF problems, as they have better accuracy in reaching the global optimum solutions [14-16]. However, the GA suffers from a severe problem which is the high computational burden.

Several optimization methods have been proposed in the literature, such as particle swarm optimization (PSO) [17], biogeography-based optimization (BBO) [18], imperialist competitive algorithm [19], grey wolf optimization (GWO) [20], PSO-gravitational search optimization [21-24], differential search algorithm [25], multiphase search optimization [26], stud krill herd optimization [27], fuzzy-based PSO algorithm [28], chaotic self-adaptive differential harmony search algorithm [29], blackhole-based optimization [30], multi-objective harmony search algorithm [31], Jaya algorithm [32], teaching learning-based algorithm [33], biography-based predator-prey technique [34], differential evolution algorithm [5], artificial bee colony (ABC) [35], simple distributed algorithm [36], firefly algorithm (FA) [3] and other several on deterministic and hybrid methods [37]. Although these methods have better performance than classical methods, some may fail to get the optimum global settings for the control values. Additionally, some of them suffer from the problem of high computational burdens.

In this paper, different new meta-heuristic optimization algorithms are presented to solve the OPF problem. The objective function of the OPF is designed so that it has a multi-objective instead of having a single objective. The objectives intended in this paper are reducing fuel costs, transmission power losses, and voltage drops. The proposed methods are tested using MATLAB simulations over the IEEE 30-bus test system. The performance of the proposed methods was assessed and evaluated in terms of accuracy and computational burden. 
The rest of this paper is organized as follows: Section 2 shows the mathematical modeling of the OPF objective function, including its relative constraints. Section 3 presents the proposed methods for solving the OPF problem. Four case studies are illustrated in Section 4 , including a detailed analysis.

\section{The Mathematical Modeling of OPF Objective Function}

The OPF equation consists of two main vectors: the first is the input vector, which contains the parameters that are tuned so that OPF is achieved. The second vector is the output, formed from all parameters dependent on the input vector. The input vector is expressed as

$$
x=\left[P_{G 2} \ldots P_{G N G}, V_{G 1} \ldots V_{G N G}, T_{1} \ldots T_{N T}, Q_{C 1} \ldots Q_{C N C}\right]
$$

where NG is the number of generators, NT is the number of transformers, and NC is the number of reactive power compensation units. $P_{G i}$ is the setting of the output power of the generator connected to bus $i$. $T_{i}$ is the transformer tap setting of the transformer connected to bus $i$. $Q_{C i}$ is the reactive power value of the reactive power compensation unit connected to bus $i$. It is essential to mention that in the case of the output power of the generator, $P_{G 1}$ is the output power of the slack bus, so it is not included in the input vector. Instead, since it is dependent on the input vector. This is why the number of the bus of the output power in the input vector starts from bus 2 . Regarding the input vector, it is formed according to Equation (2):

$$
y=\left[P_{G 1}, V_{L 1} \ldots V_{L N L}, Q_{G 1} \ldots Q_{G N G}, S_{l 1} \ldots S_{l N T L}\right]
$$

where $N L$ is the number of load buses, NTL is the number of transmission lines, $V_{L i}$ is the load voltage of bus $i$. $Q_{G i}$ is the reactive power of the generator connected to bus $i$ and $S_{l i}$ is the load flowing in transmission line $i$. To understand the power flow equations, firstly, it is required to build the admittance matrix as per Equation (3):

$$
\left[\begin{array}{c}
I_{1} \\
I_{2} \\
\vdots \\
I_{i}
\end{array}\right]=\left[\begin{array}{cccc}
Y_{11} & Y_{12} & \ldots & Y_{1 j} \\
Y_{21} & Y_{22} & \ldots & Y_{2 j} \\
\vdots & \ddots & \ldots & \vdots \\
Y_{i 1} & Y_{i 2} & \ldots & Y_{i j}
\end{array}\right]\left[\begin{array}{c}
V_{1} \\
V_{2} \\
\vdots \\
V_{i}
\end{array}\right]
$$

where $I_{i}$ is the current flowing through bus $I, V_{i}$ is the voltage of bus $i$ and $Y_{i j}$ is the admittance element of the $i_{t h}$ row and $j_{t h}$ column. According to the load flow equations, the total generated power must equal the total power of the load and the power losses as shown in Equations (4) and (5):

$$
\begin{aligned}
& P_{G i}-P_{D i}-V_{i} \sum_{j=1}^{N B} V_{j}\left(G_{i j} \cos \theta_{i j}+B_{i j} \sin \theta_{i j}\right)=0 \\
& Q_{G i}-Q_{D i}-V_{i} \sum_{j=1}^{N B} V_{j}\left(G_{i j} \sin \theta_{i j}+B_{i j} \cos \theta_{i j}\right)=0
\end{aligned}
$$

where $P_{D i}$ and $Q_{D i}$ are the active and reactive power demand of the load connected to bus $i, \theta_{i j}$ is the voltage angle between buses $i$ and $j . G_{i j}$ and $B_{i j}$ represent the real and imaginary parts of the admittance matrix, respectively.

Although the power flow equations shown in Equations (4) and (5) look simple, the optimization of these equations is sophisticated due to the increased number of constraints due to the operation limits for the bus voltages and active and reactive power limits. The minimum and maximum limits for control variables in Equation (1) and the corresponding dependent variables in Equation (2) can be expressed as follows:

$$
\begin{aligned}
V_{L i}^{m i n} & \leq V_{L i} \leq V_{L i}^{m a x}, i=1, \ldots, N L \\
Q_{G i}^{m i n} & \leq Q_{G i} \leq Q_{G i}^{m a x}, i=1, \ldots, N G
\end{aligned}
$$




$$
\begin{gathered}
S_{l i} \leq V_{l i}^{\max }, i=1, \ldots, N T L \\
P_{G i}^{\text {min }} \leq P_{G i} \leq P_{G i}^{\text {max }}, i=2, \ldots, N G \\
V_{G i}^{\text {min }} \leq V_{G i} \leq V_{G i}^{\max }, i=1, \ldots, N G \\
T_{i}^{\text {min }} \leq T_{i} \leq T_{i}^{\max }, i=1, \ldots, N T \\
Q_{C i}^{\text {min }} \leq Q_{C i} \leq Q_{C i}^{\max }, i=1, \ldots, N C
\end{gathered}
$$

Now, the OPF equation can be formed as per Equation (13):

$$
\begin{aligned}
F_{p}(x, y)=F(x, y) & +\lambda_{p}\left(P_{G l}-P_{G l}^{l i m}\right)^{2}+\lambda_{v} \sum_{i=1}^{N L}\left(V_{L i}-V_{L i}^{l i m}\right)^{2} \\
& +\lambda_{Q} \sum_{i=1}^{N G}\left(Q_{G i}-Q_{G i}^{l i m}\right)^{2}+\lambda_{s} \sum_{i=1}^{N T L}\left(S_{l i}-S_{l i}^{l i m}\right)^{2}
\end{aligned}
$$

where $\lambda_{p}, \lambda_{v}, \lambda_{Q}$ and $\lambda_{s}$ are the penalty factors applied to keep the active power of the slack bus, bus load voltages, reactive power generation, and branch complex powers within acceptable limits. $P_{G l}^{\lim }, V_{L i}^{l i m}, Q_{G i}^{l i m}$ and $S_{l i}^{l i m}$ are the limit values for the active power of the slack bus, bus load voltages, reactive power generation, and branch complex powers. Equations (14)-(17) represent the mathematical formulation of these limits:

$$
\begin{gathered}
P_{G s l}^{l i m}= \begin{cases}P_{G s l}^{\min }, & P_{G s l}<P_{G s l}^{\min } \\
P_{G s l}^{\max } & P_{G s l}>P_{G s l}^{\max } \\
P_{G s l} & P_{G s l}^{\min } \leq P_{G s l} \leq P_{G s l}^{\max }\end{cases} \\
V_{L i}^{l i m}= \begin{cases}V_{L i}^{\min }, & V_{L i}<V_{L i}^{\min } \\
V_{L i}^{\max } & V_{L i}>V_{L i}^{\max } \\
V_{L i} & V_{L i}^{\min } \leq V_{L i} \leq V_{L i}^{\max }\end{cases} \\
Q_{G i}^{l i m}= \begin{cases}Q_{G i}^{\min }, & Q_{G i}<Q_{G i}^{\min } \\
Q_{G i}^{\max } & Q_{G i}>Q_{G i}^{\max } \\
Q_{G i} & Q_{G i}^{\min } \leq Q_{G i} \leq Q_{G i}^{\max }\end{cases} \\
S_{l i}^{l i m}= \begin{cases}S_{l i}^{\max } & S_{l i}>S_{l \max } \\
S_{l i} & S_{l i} \leq S_{l i}^{\max }\end{cases}
\end{gathered}
$$

As mentioned before, there are three main objectives for the optimization of $\mathrm{OPF}$, as shown in the following list:

1. The reduction in fuel costs;

2. The reduction in the power transmission losses;

3. The reduction in voltage drops.

To minimize the consumption of generator fuel, the following objective function is applied:

$$
\min _{x} F(x, y)=\min _{x} \sum_{i=1}^{N G}\left(a_{i}+b_{i} P_{G i}+c_{i} P_{G i}^{2}\right)
$$

where $a_{i}, b_{i}$ and $c_{i}$ are the cost factors of the generator cost function.

In the case of reducing the power losses, the objective function can be expressed as

$$
\min _{x} F(x, y)=\min _{x} \sum_{j=1}^{N T L} \sum_{i=1}^{N L} g_{i j}\left[V_{i}^{2}+V_{j}^{2}-2 V_{i} V_{j} \cos \left(\theta_{i}-\theta_{j}\right)\right]
$$

where $g_{i j}$ is the conductance value of the transmission line between buses $i$ and $j . V_{i}$ and $\theta_{i}$ are the voltage magnitude and angle of bus $i$ while $V_{j}$ and $\theta_{j}$ are the voltage magnitude and angle of bus $j$. 
If the purpose of the OPF optimization is to reduce the voltage drop at the transmission lines, then the objective function is set as per Equation (20):

$$
\min _{x} F(x, y)=\min _{x} \sum_{j=1}^{N T L}\left|V_{i}-V_{i}^{r e f}\right|
$$

where $V_{i}^{r e f}$ is the reference value of voltage magnitude at bus $i$.

Since the three objectives mentioned above are all critical, combining two or more objectives in the same objective function is possible. In this paper, a multi-objective function for achieving the three objectives is used as per Equation (21):

$$
\min _{x} F(x, y)=\min _{x} \sum_{i=1}^{N G}\left(a_{i}+b_{i} P_{G i}+c_{i} P_{G i}^{2}\right)+\sum_{j=1}^{N T L} \sum_{i=1}^{N L} g_{i j}\left[V_{i}^{2}+V_{j}^{2}-2 V_{i} V_{j} \cos \left(\theta_{i}-\theta_{j}\right)\right]+\sum_{j=1}^{N T L}\left|V_{i}-V_{i}^{r e f}\right|
$$

\section{The Proposed Meta-Heuristic Algorithms for Solving the OPF Problem}

As mentioned before, in this paper, three meta-heuristic optimization algorithms are used to optimize the solution of the OPF problem, as shown in the following subsections.

\subsection{The Multi-Verse Optimization Algorithm}

There are three main elements behind the multi-verse theory, which create the very first idea of the MVO. The first element was unobservable until now, which is the white hole; it occurs at the creation of a universe or the collision of two neighboring universes. The following element is the opposite of the first one in its behavior and is called the black hole. We can always observe black holes, and they are characterized by the vast gravitational forces that make every ambient object attracted to them. The last element is called the wormhole; it has the authority to exchange objects either between different universes or between different parts of one universe [38,39].

Additionally, the idea of the universe expansion process is clarified by the multi-verse theory, which depends mainly on the inflation rate. The universe elements are formed, and they are controlled by that rate. We can achieve a stable phase between two parallel universes by separating the three elements, white, black, and wormholes. This process is exactly like the MVO search process.

The above method has been applied in significant optimization applications and the management of processes related to renewable energy and power systems $[38,39]$.

Figure 1 shows the main idea of the MVO, we have $n$ universes, and each of them reflects a solution. The wormhole is the path of the objects from a high inflation rate universe to other lower inflation rate universes. The optimum case is when the universe can receive objects from all other universes, which means that it has the lowest inflation rate.

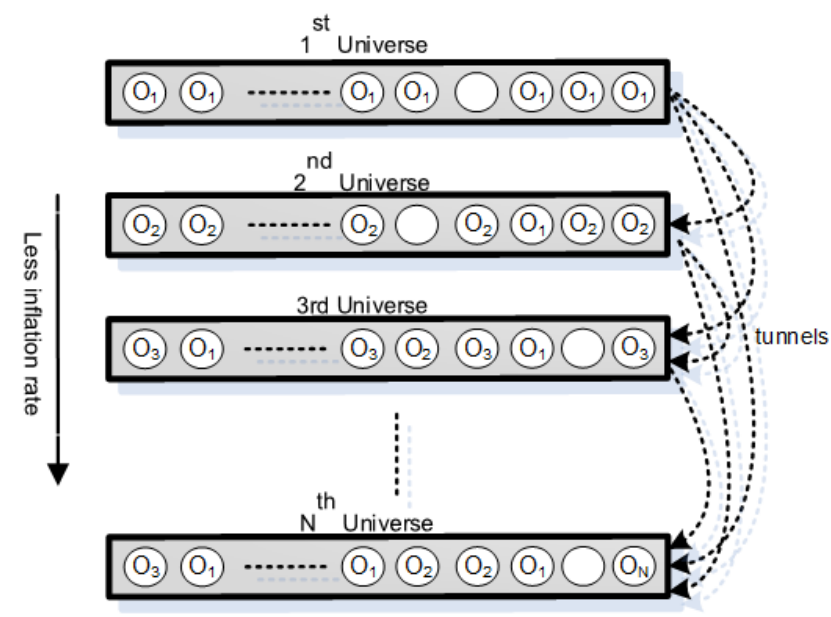

Figure 1. Theory behind multi-verse optimization (MVO) algorithm. 
To mathematically model the MVO algorithm, in Equation (22), there is an illustration for the roulette wheel mechanisms that can randomly arrange all universes, assuming that $d$ is the number of variables and $n$ is the number of candidate solutions [40-42]:

$$
U=\left[\begin{array}{cccc}
x_{1}^{1} & x_{1}^{2} & \cdots & x_{1}^{d} \\
x_{2}^{1} & x_{2}^{2} & \cdots & x_{2}^{d} \\
\vdots & \vdots & \vdots & \vdots \\
x_{n}^{1} & x_{n}^{2} & \cdots & x_{n}^{d}
\end{array}\right]
$$

where $x_{i}^{j}$ is the $j^{\text {th }}$ parameter of the $i^{\text {th }}$ universe. Each parameter can be calculated from Equation (23):

$$
x_{i}^{j}= \begin{cases}x_{k^{\prime}}^{j} & r_{1}<N I\left(U_{i}\right) \\ x_{i}^{j}, & r_{1} \geq N I\left(U_{i}\right)\end{cases}
$$

where $x_{k}^{j}$ is the $j^{\text {th }}$ parameter of the $k^{\text {th }}$ universe, $r_{1}$ is a random binary number which can be either 0 or 1 , and $\operatorname{NI}\left(U_{i}\right)$ is the inflation rate of the $i^{\text {th }}$ universe. As shown in Equation (23), white holes are formed with different inflation rates using the roulette wheel mechanism. As we said, lower inflation universes can receive more objects through white/black holes. However, another mechanism describes more improvements in the inflation rate made by each universe using wormholes. This process can be shown as per Equation (24):

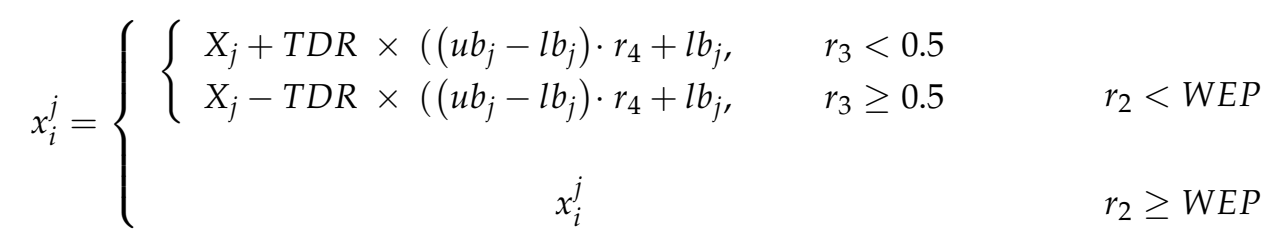

where $X_{j}$ is the $j^{\text {th }}$ element of the best solution (best universe created). $l b_{j}$ and $u b_{j}$ are the lower and upper bounds of this element. $r_{2}, r_{3}$ and $r_{4}$ are binary numbers. WEP is the wormhole existence probability while the TDR is traveling wave distance. As we go for more iterations, we observe the linearity of the increasing WEP which confirms the progress of the optimization algorithm and to what extent it can achieve the best solution. The WEP is updated based on the adaptive equation as described in Equation (25):

$$
W E P=\min +l \times\left(\frac{\max -\min }{L}\right)
$$

where min and max are the boundaries for the WEP coefficient; $l$ is the order of the iteration, while $L$ is the maximum number of iterations.

As per Equation (12), this adaptive formula is used to update $T D R$, which acts similarly to the WEP; when the number of iterations increases, the value of the TDR increases, which guarantees a more precise local search in the path to find the best solution:

$$
T D R=1-\left(l^{\frac{1}{p}} / L^{\frac{1}{p}}\right)
$$

where $p$ is the coefficient that controls the accuracy and speed of algorithm convergence, Figure 2 shows the flow chart of the MVO algorithm. 


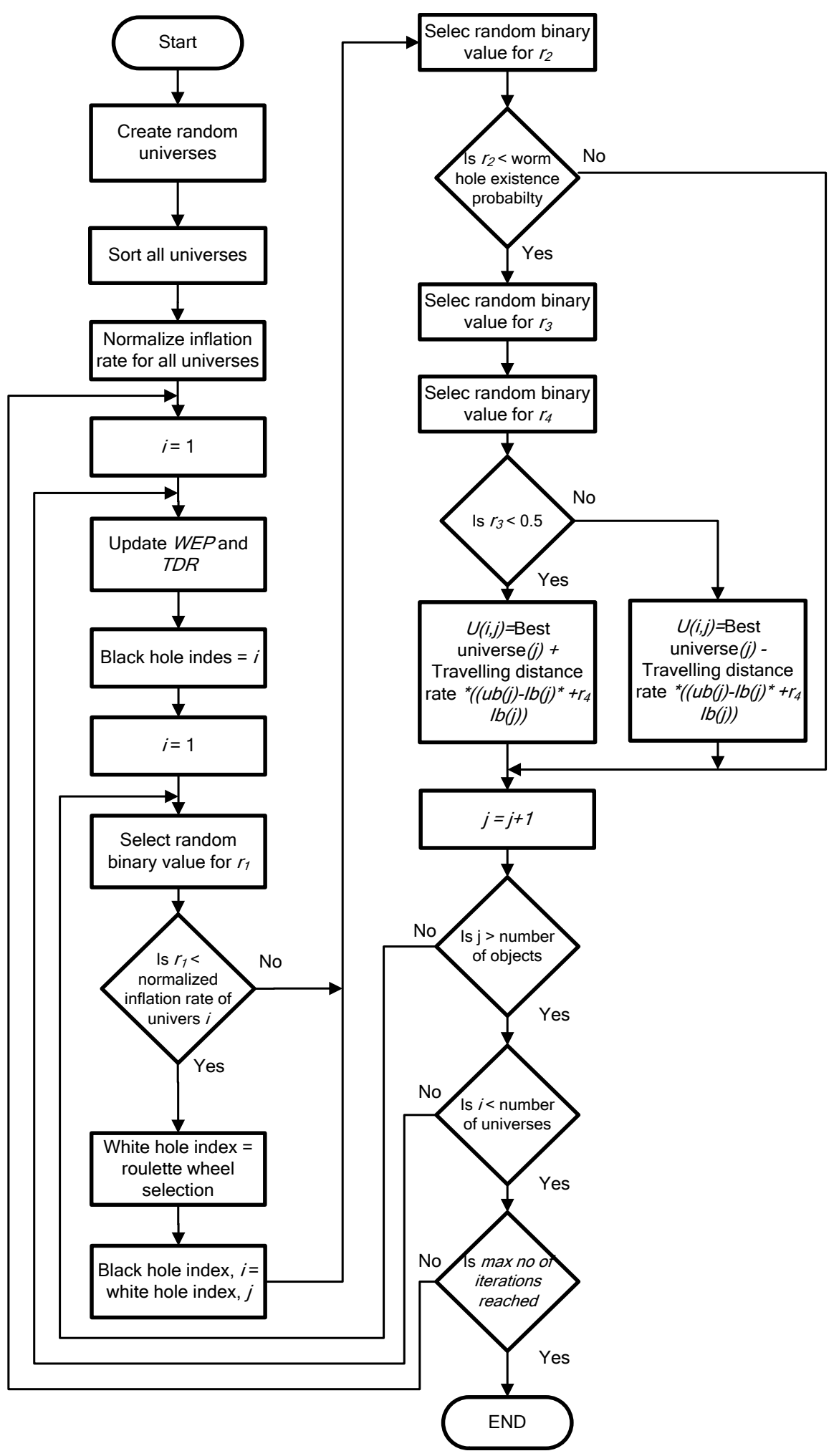

Figure 2. The flowchart of the MVO algorithm.

\subsection{The Grasshopper Optimization Algorithm}

Grasshoppers are harmful insects. They are known for their harmful effect of reducing agriculture production. Figure 3 shows the change that occurs when grasshoppers travel and join a big group, among other creatures, despite these usually being seen individually $[40,41]$. The group size is big enough to be terrifying to ranchers. This behavior is seen in both the nymph and the adulthood, which makes them extraordinary [40,41]. Nymph 
grasshoppers gather in massive numbers, bouncing and moving like trigger clambers, eating their way through the harvest. After this stage, when they have grown into adults, they build a multitude of structures which are noticeable all around. This phase is the evacuation process of the grasshoppers.

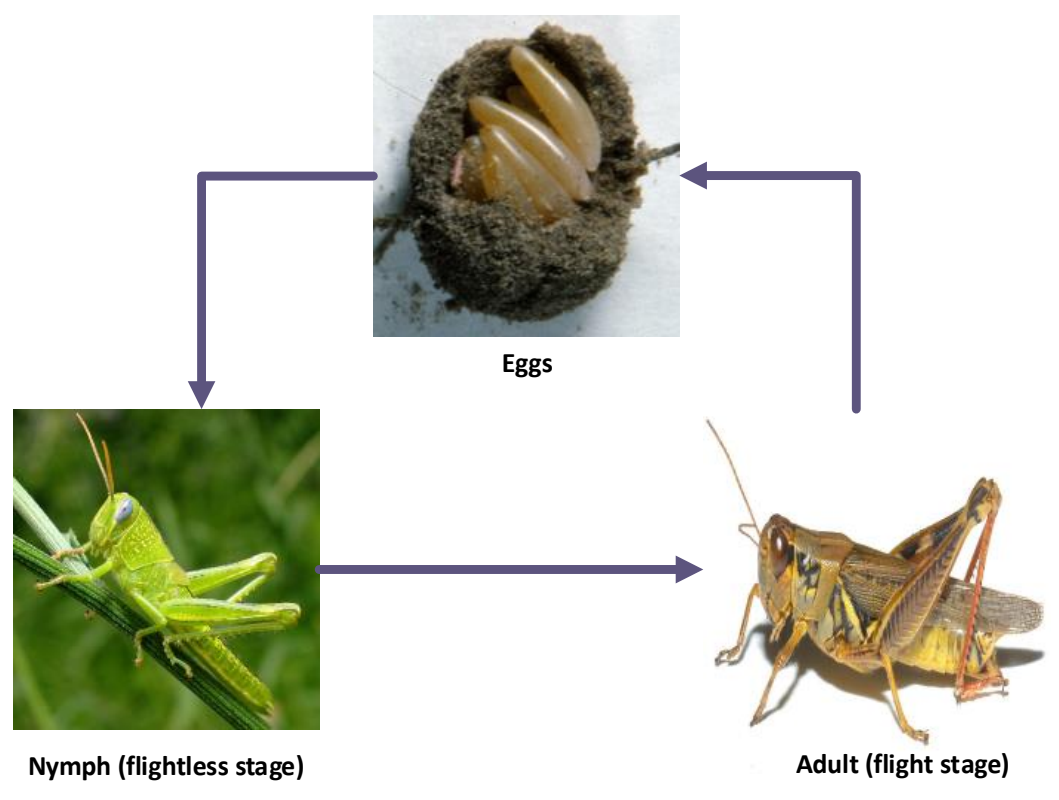

Figure 3. The life cycle of grasshoppers.

In the icteric stage, the main characteristic of the herd is the moderate activity and little strides of the grasshopper. On the other hand, investigation for a food source and sudden movement among extended zones is the herd's most crucial ability. The natural inspired techniques categorize two denominations. The first denomination is exploration; in this denomination, the observation of the movement of the search agents is an unexpected motion. Subsequently, we need a mathematical model for the swarm behavior to accomplish the design of the developed inspired technique. The mathematical model of the swarming behavior of the grasshopper is presented as follows $[40,41]$ :

$$
X_{n}=S_{n}+G_{n}+A_{n}
$$

where $X_{n}$ is the current placement of any of the grasshoppers, $S_{n}$ is the social interaction, $G_{n}$ is the gravity force, and $A_{n}$ shows the wind advection. An imprecise attitude of the herd can be evaluated from the following equation:

$$
X_{n}=r_{1} S_{n}+r_{2} G_{n}+r_{3} A_{n}
$$

where $r_{1}, r_{2}$, and $r_{3}$ are random numbers from [0,1].

The social interaction $\left(S_{n}\right)$ is obtained through the following equation:

$$
S_{n}=\sum_{\substack{m=1 \\ m \neq n}}^{N} s(d n m) \hat{d_{m n}}
$$

where $(\mathrm{dnm})$ is the displacement between two nearby grasshoppers, and it is evaluated from $d n m=\left|X_{m}-X_{n}\right| ;(s)$ is the function that indicates the vigor of social forces and is calculated as follows:

$$
\mathrm{s}(\mathrm{r})=\mathrm{f} e^{\frac{-r}{l}}-e^{-r}
$$


Additionally, the $\left(\hat{d_{m n}}\right)$ is a vector in which its magnitude equals one displacement and two grasshoppers and be obtained as follows:

$$
\hat{d_{n m}}=\frac{x_{m-x_{n}}}{d_{n m}}
$$

where (f) represents the attraction vigor while (l) is the level of the attractive longitude. Figure 4 illustrates the effect of the value of (s) on the grasshopper's social interaction. Additionally, it is shown in Figure 4a that the repulsion between grasshoppers befalls an interval of 0-2.079 with displacement changing from 0 to 15 . A comfort zone occurs when the distance between two grasshoppers is equal to 2.079 units, making the attraction and repulsion between them vanish. In Figure 5, the variation of the two factors $(l, f)$ is considered in plotting the value of (s) in Equation (30). It is noticeable that for a few values of 1 and $f$, for example, 1, 0.5 , respectively, both the attraction and repulsion zones are microscopic. In Figure 6, the relationship between grasshoppers' interaction and the comfort zone is expressed by the value of (s) $[40,41]$. There is a prominent issue in implementing the value of (s) when applying strong forces between individual grasshoppers. Despite being able to determine the attraction and repulsion zones, its value reaches zero with an extended displacement of more than 10 . The force of gravity is evaluated from the following:

$$
G_{n}=-\mathrm{g} \hat{e}_{g}
$$

where $(\mathrm{g})$ is the gravitational constant and $\left(\hat{e_{g}}\right)$ shows a unity factor towards the center of the earth. The $(A)$ component in Equation (27) is calculated as follows:

$$
A_{n}=\mathrm{u} \hat{e_{w}}
$$

where $(\mathrm{u})$ is a constant drift and $\left(\hat{e_{w}}\right)$ is a unit vector in the direction of the wind. As illustrated in Figure 3, the wind direction has a significant effect on the nymph grasshoppers as they have no wings. By replacing $S, G$, and $A$ in Equation (27), the equation can be expressed as follows:

$$
X_{i}=\sum_{\substack{m=1 \\ m \neq n}}^{N} s\left(\left|x_{m}-x_{n}\right|\right) \frac{x_{m}-x_{n}}{d_{n m}}-\mathrm{ge} \hat{g}_{g}+\mathbf{u} \hat{e_{w}}
$$

where $N$ is the number of grasshoppers.

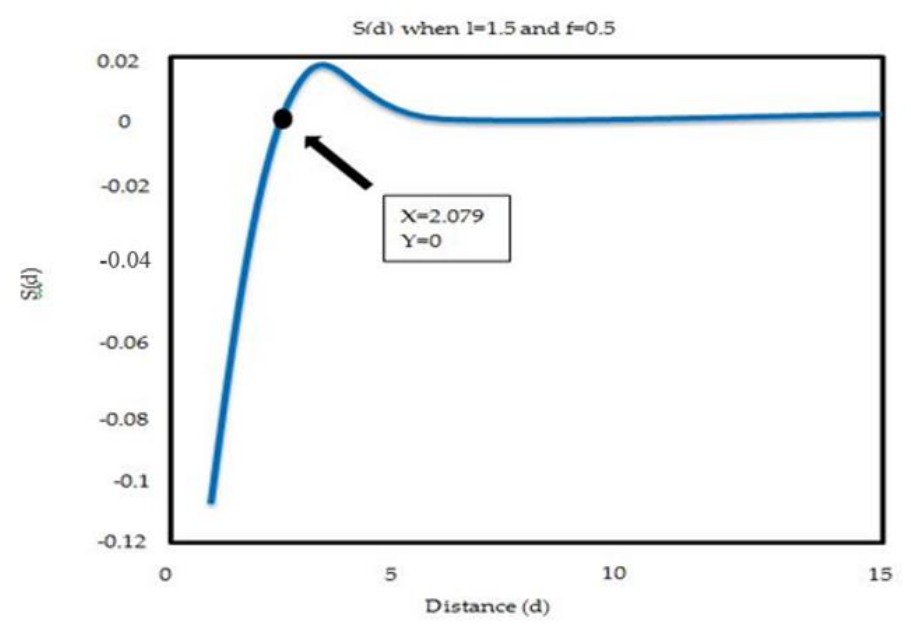

(a)

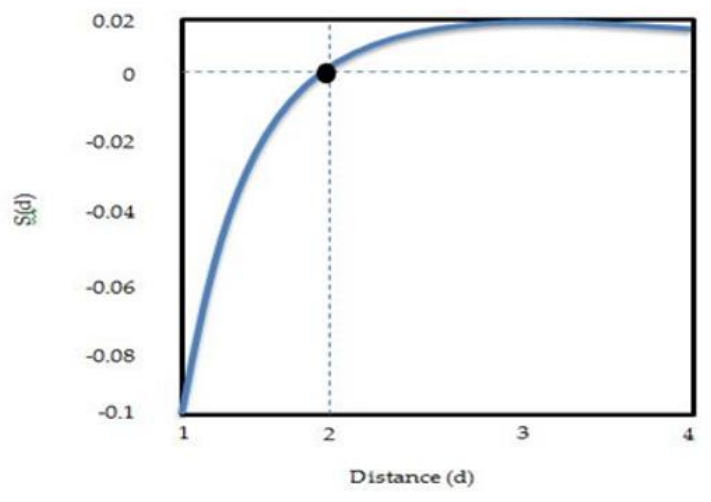

(b)

Figure 4. (a) Value of (s) at $1=1.5$ and $\mathrm{f}=0.5$; (b) zone of the value of (s) as displacement extends from 1 to 4. 
$\mathrm{l}=0.5$

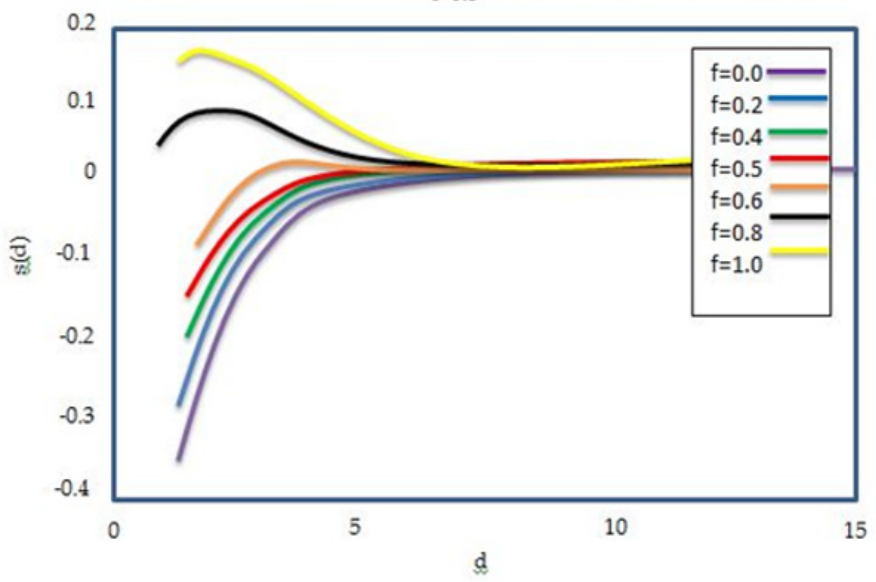

$\mathrm{f}=0$.

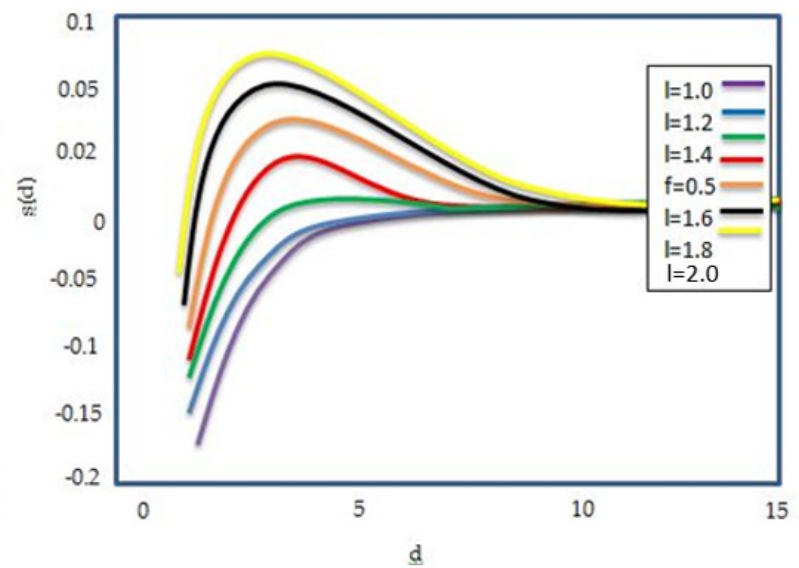

Figure 5. Effectiveness of the value of (s) as it changes with one of the following $l$.

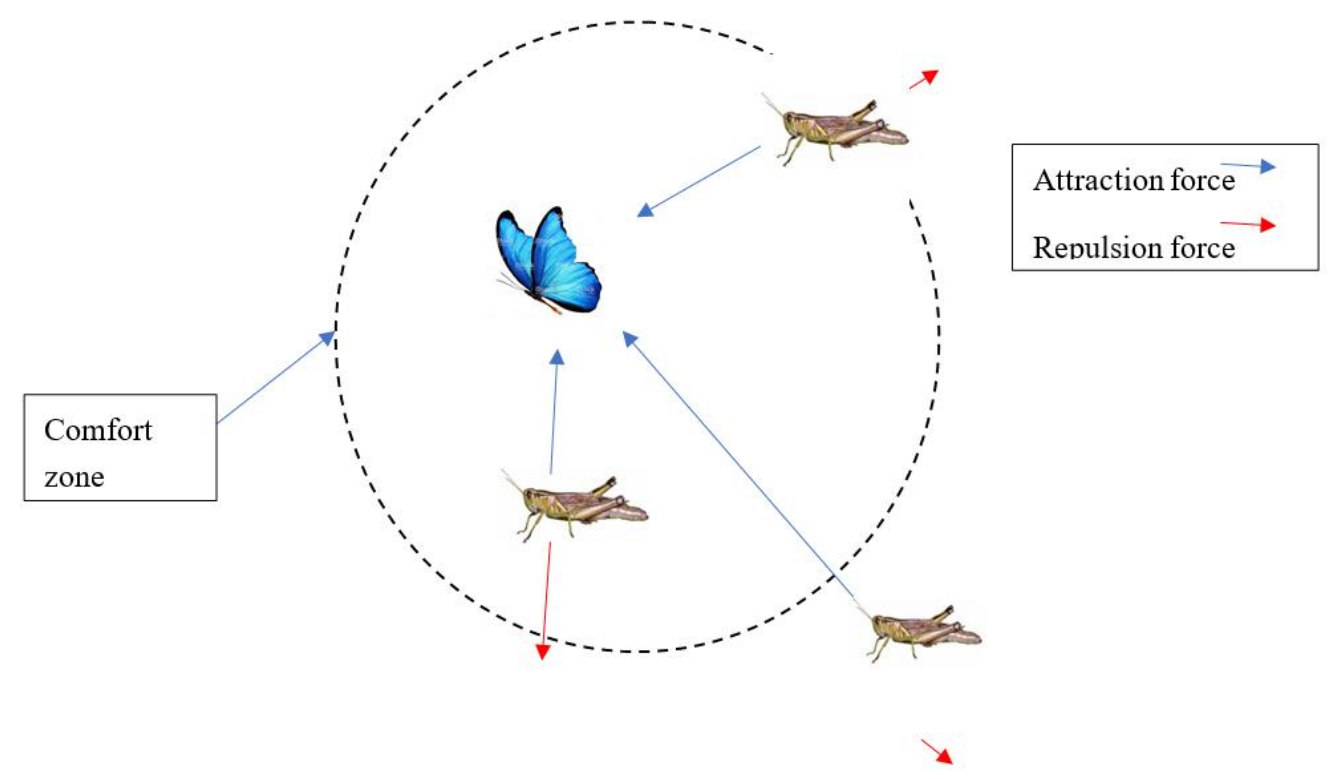

Figure 6. The embryonic reformist styles between each grasshopper in the herd.

At the point at which there is a change in the program's execution, the minor female of the grasshoppers is not allowed to reach that location, although these grasshoppers can land on the ground. As a result, in this case, the equation of the entire simulation blocked the algorithm from both the exploration and exploitation of a search agent around the solution, so it is never used. In conclusion, the implemented scheme of the herd took place in the free space. According to Equation (34), the interaction between the grasshoppers and each of the others in the swarm is implemented. However, when it comes to solving optimization problems, this mathematical model cannot be directly used because the grasshoppers reaches the comfort zone quickly, and the herd does not focus on a particular nearby point. Therefore, Equation (34) is modified and proposed as follows to solve the optimization problems:

$$
X_{m}^{d}=\mathrm{c}\left[\sum_{\substack{m \neq n \\ m \neq n}}^{N} c \frac{u b_{d}-l b_{d}}{2} * \mathrm{~s}\left(\left|x_{m}^{d}-x_{n}^{d}\right|\right)\left(\frac{x_{m}-x_{n}}{d_{n m}}\right)\right]+\hat{T_{d}}
$$

where $u b_{d}$ and $l b_{d}$ are the upper and lower bounds in the D-th dimensions, respectively. $\hat{T_{d}}$ is the optimal solution so far, and (c) is a decreasing degree to contract the attraction 
zone, repulsion zone, and comfort zone. The $(\mathrm{G})$ component is neglected, and the wind direction is assumed to be always towards the target $\left(T_{d}\right)$. As it appears in Equation (35), the following displacement of a given grasshopper is determined by three factors. These factors are the current displacement of that given grasshopper, its objective displacement, and the locations of the other grasshoppers. This technique is different from that of PSO, as we mentioned before in the literature.

The position and the velocity vectors are two critical factors that are needed to define each particle in the particle swarm optimization (PSO), while there is only one vectorthat is required to define the search agent in the grasshopper optimization (GOA).

Other factors differentiate between both techniques in determining the displacement of particles. According to the PSO, the essential factors in locating the position of particles are the current displacement, the best solution obtained by an individual, and the best solution obtained by the swarm. At the same time, concerning the GOA, it is the current location, the superior solution gained by an individual, the best solution obtained by the swarm, and the locations of the other search agents. According to Equation (35), it is clear that the adaptive element (c) has repeated two times for the following reasons:

1. The first (c) is nearly comparable to the inertial weight (w) in PSO, and it is responsible for the remission of the motion of grasshoppers towards its target, which occurs by managing both exploration and exploitation.

2. The second (c) aims to reduce the attraction, repulsion, and comfort zones between the grasshoppers.

With respect to Equation (35), it is evident that the element (c) inside the equation is directly proportional to the number of iterations as it participates in reducing the attraction and repulsion between the grasshoppers. Additionally, the outer element (c) plays a role in decreasing the concourse towards the target by increasing the number of iterations.

Finally, according to Equation (35), the start of this equation represents the location of the other grasshoppers and simulates their interaction in nature. Additionally, the second part which is identified by $\left(\hat{T_{d}}\right)$ simulates its motion capability towards the target.

Generally, as in the icteric phase, when grasshoppers have no wings, they tend to stir and look locally for their food; in the next stage, they learn to move freely in the air as they explore much larger level zones.

In stochastic optimization techniques, finding promising regions of the search space is essential, and thus exploration is essential. After finding these regions, exploitation search agents search locally to find the global optimum as an accurate approximation value. The coefficient (c) is calculated as follows:

$$
\mathrm{c}=\operatorname{cmax}-\mathrm{i}\left(\frac{\operatorname{cmax}-c \min }{I}\right)
$$

where cmax and cmin are the limitation values, (i) indicates the present iteration, while $(I)$ is the ultimate number of iterations. In this work, cmax and cmin are set to be 1 and 0.00004 , respectively. In reality, the global optimum solution is unknown, so there is no target to achieve it. Therefore, there must be a clear objective for grasshoppers in each step as to which is the best objective value. This will help GOA to keep the most objective value in the search space in each iteration and require grasshoppers to move towards it.

The flowchart of the grasshopper optimization technique is expressed in Figure 7. The GOA starts the optimization by initializing the behavior parameters such as $S_{n}, G_{n}, A_{n}$, cmax, cmin, etc., then generating random solutions. Additionally, the fitness function is evaluated, leading to updating the locations of search agents based on Equation (33). The best target position was obtained and updated in each iteration. After that, the number of iterations is compared with the population size, and if the number of iterations is greater than the population size, then the best position will be observed if it reaches the best position. If not, the fitness function will be re-evaluated. Therefore, if the best position is achieved, it will be assigned to the senior position, and if not, the fitness function will be 
evaluated. Finally, the location and the objective of the best target are returned as the best approximation for the global optimum.

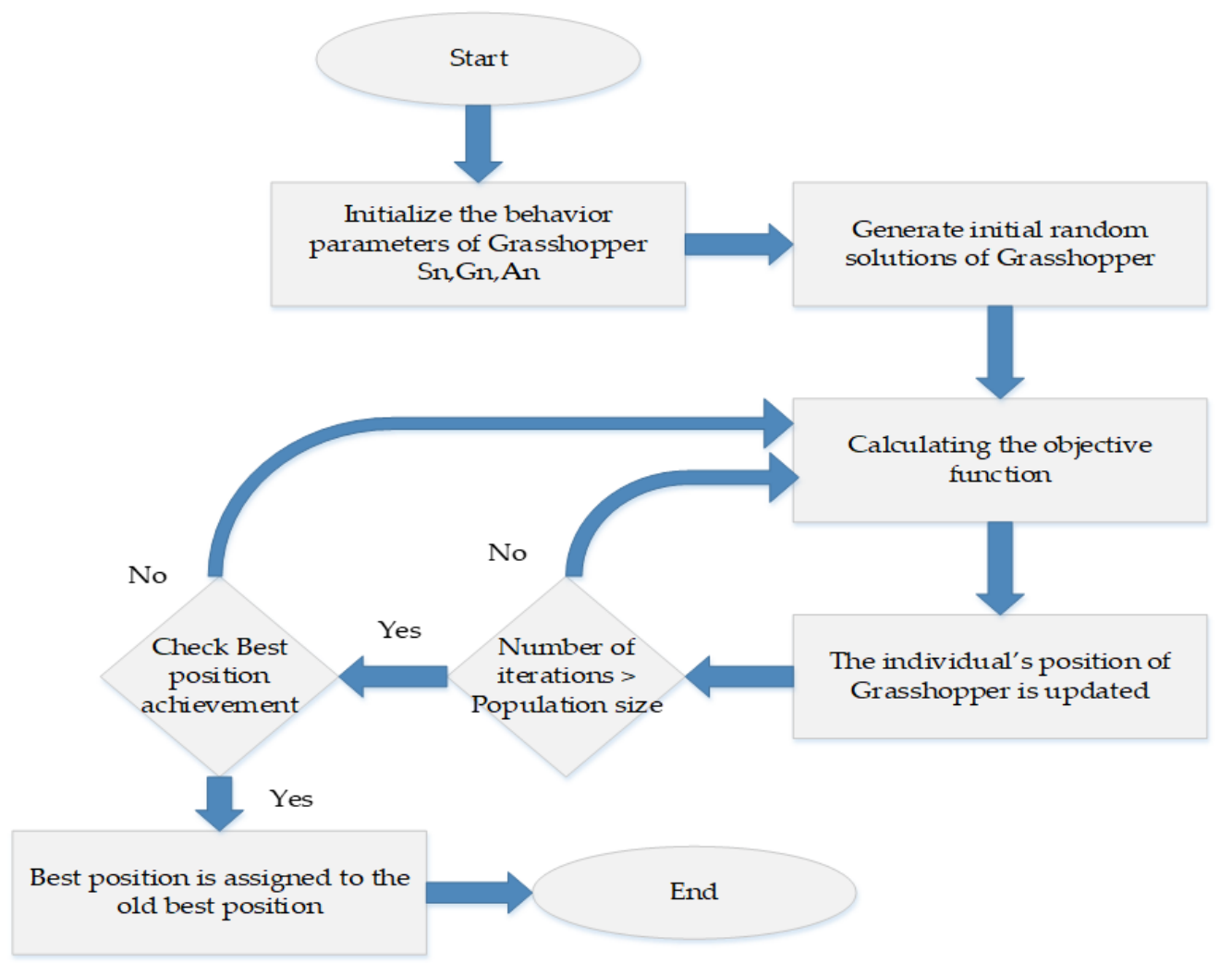

Figure 7. Flow chart of grasshopper.

\subsection{The Harris Hawks Optimization Algorithm}

Harris hawks optimization is a metaheuristic optimization proposed in [42] that is inspired by the cooperative behavior of Harris hawks in hunting, chasing, and besieging their victims. The $\mathrm{HHO}$ is based on population optimization without having any gradients, which gives it a competitive edge over other techniques in terms of conversion speed.

The HHO consists of two main phases: exploration and exploitation. Additionally, there is a transition phase through which the algorithm is switched from exploration to exploitation.

In the exploration phase, Harris hawks start to search randomly for victims as per the following equation:

$$
X(t+1)=\left\{\begin{array}{cc}
X_{\text {rand }}(t)-r_{1}\left|X_{\text {rand }}(t)-2 r_{2} X(t)\right| & q \geq 0.5 \\
\left(X_{\text {rabbit }}(t)-X_{m}(t)\right)-r_{3}\left(L B+r_{4}(U B-L B)\right) & q<0.5
\end{array}\right.
$$

where $X(t+1)$ is the location of the hawks in the iteration $(t+1)$; $X_{\text {rabbit }}(t)$ is the location of the rabbit (the victim); $r_{1}$ to $r_{4}$ and $q$ are random numbers that can vary between 0 and 1 ; $X_{\text {rand }}(t)$ represents a hawk which is chosen randomly; and $X_{m}$ is the average location of the current population of hawks which can be calculated from Equation (38):

$$
X_{m}(t)=\frac{1}{N} \sum_{i=1}^{N} X_{i}(t)
$$

where $X_{i}(t)$ indicates the position of each hawk at iteration $t$ while $N$ represents the total number of hawks. 
As mentioned above, after finishing the exploration stage, there is a transient stage before moving to the exploitation stage. At this transient stage, it is necessary to model the energy of the rabbit as per Equation (39):

$$
E=2 E_{0}\left(1-\frac{t}{T}\right)
$$

where $E$ is the escaping energy of the rabbit, $T$ is the maximum number of iterations and $E_{0}$ is the initial state of the rabbit energy. The value of $E_{0}$ is varies between -1 and 1 based on the physical fitness of the victim. When $E_{0}$ goes towards -1 , this means that the victim is losing its energy and vice versa.

According to the behavior of rabbits, the relation between the rabbit energy and the time is inversely proportional. This means that as long as $t$ increases, the $E$ is decreased. Additionally, based on $E$, Harris hawks decide to either search different areas to detect the location of the rabbit when $|E| \geq 1$, or move forward to the exploitation phase.

In the exploitation phase, two behaviors need to be modeled. The first is the soft besiege in which the rabbit energy is still high and can run fast; in this condition, Harris hawks try to softly follow and put it under surveillance until it starts to get exhausted. The second is the hard besiege; the prey in this behavior is tired and does not have sufficient energy to escape. As a result, the Harris hawks in this mode form closed circles to make a sudden attack. Figure 8 shows Harris hawk attack patterns.

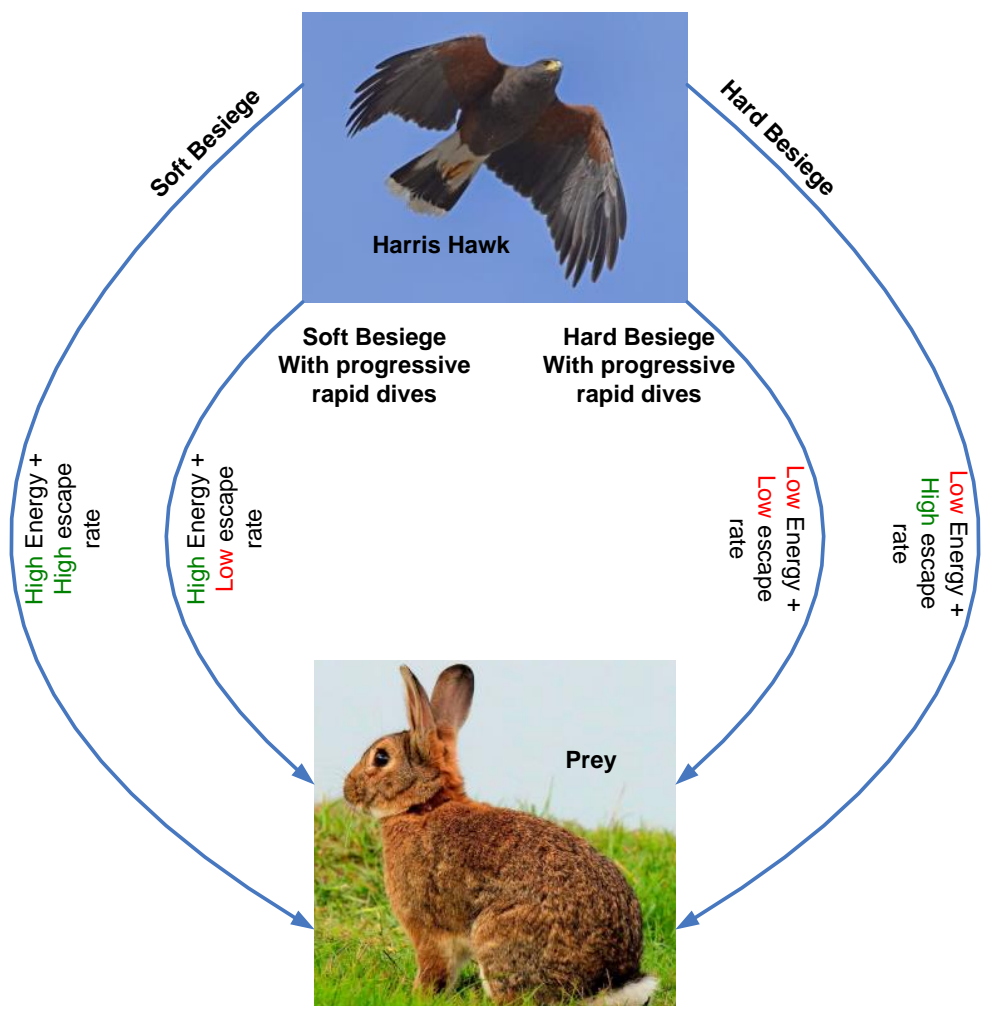

Figure 8. Harris hawk prey attack patterns.

To mathematically model the two behaviors, let $r$ be the percentage of the successful escape of the rabbit. If $|E| \geq 0.5$ and $r \geq 0.5$, this means that the rabbit has relatively high escaping energy, and at the same time, the chance of successful escape is higher than $50 \%$. This means that the Harris hawks will perform a soft besiege and will update their location according to Equation (40):

$$
X(t+1)=\Delta X(t)-E\left|J X_{\text {rabbit }}(t)-X(t)\right|
$$


where $\Delta X(t)$ is the position difference between the rabbit and the hawks- this value can be calculated as follows:

$$
\Delta X(t)=X_{\text {rabbit }}(t)-X(t)
$$

Moreover, $J$ is a random number that represents the jump strength that can get from Equation (42) as follows:

$$
J=2\left(1-r_{5}\right)
$$

where $r_{5}$ is a random number that varies between 0 and 1 .

If $|E| \geq 0.5$ and $r<0.5$, this means that the rabbit has high energy. However, the chance of successful escape is not significant. In this case, the harris hawks will perform a soft besiege but with progressive and rapid dives. The next movement of the hawks will be updated according to:

$$
Y=X_{\text {rabbit }}(t)-E\left|J X_{\text {rabbit }}(t)-X(t)\right|
$$

The hawks then will compare the current position with the previous dive to evaluate which is better. If the previous dive is better, the hawks will use it. If not, the hawks will then apply a new dive using the levy flight $(L F)$ equation:

$$
Z=Y+S \cdot \operatorname{LF}(D)
$$

where $D$ is the problem dimension, $S$ is a random vector with a size of $1 \times D$. The $L F$ function can be calculated according to Equation (45):

$$
L F(x)=0.01\left(\frac{u \cdot \sigma}{|v|^{\frac{1}{\beta}}}\right)
$$

where $u$ and $v$ are a random number that varies between 0 and $1 . \beta$ is a constant value of 1.5. $\sigma$ which is calculated using:

$$
\sigma=\left(\frac{(1+\beta) \sin \left(\frac{\pi \beta}{2}\right)}{\frac{(1+\beta)}{2} \cdot \beta \cdot 2^{\frac{\beta-1}{2}}}\right)^{\frac{1}{\beta}}
$$

The Hariss hawks will then evaluate positions $Y$ and $Z$ and select the best position based on Equation (47):

$$
X(t+1)= \begin{cases}Y, & F(Y)<F(X(t)) \\ Z, & F(Z)<F(X(t))\end{cases}
$$

If $|E|<0.5$ and $r \geq 0.5$, this means that the rabbit has relatively low energy, but it has a moderate chance of successful escape. In this condition, the hawks will perform a hard besiege and will update their equation based on Equation (48):

$$
X(t+1)=X_{\text {rabbit }}(t)-E|\Delta X(t)|
$$

If $|E|<0.5$ and $r<0.5$, this means that the victim has low energy and also has a low chance to escape. In this situation, the hawks will also perform a hard besiege but with progressive rapid dives at which the next position of the hawks will be updated using Equation (21). $Z$ will be calculated from Equation (18), and $Y$ will be calculated using Equation (49) as follows:

$$
Y=X_{\text {rabbit }}(t)-E\left|J X_{\text {rabbit }}(t)-X_{m}(t)\right|
$$

Figure 9 shows a flowchart of the proposed HHO. 


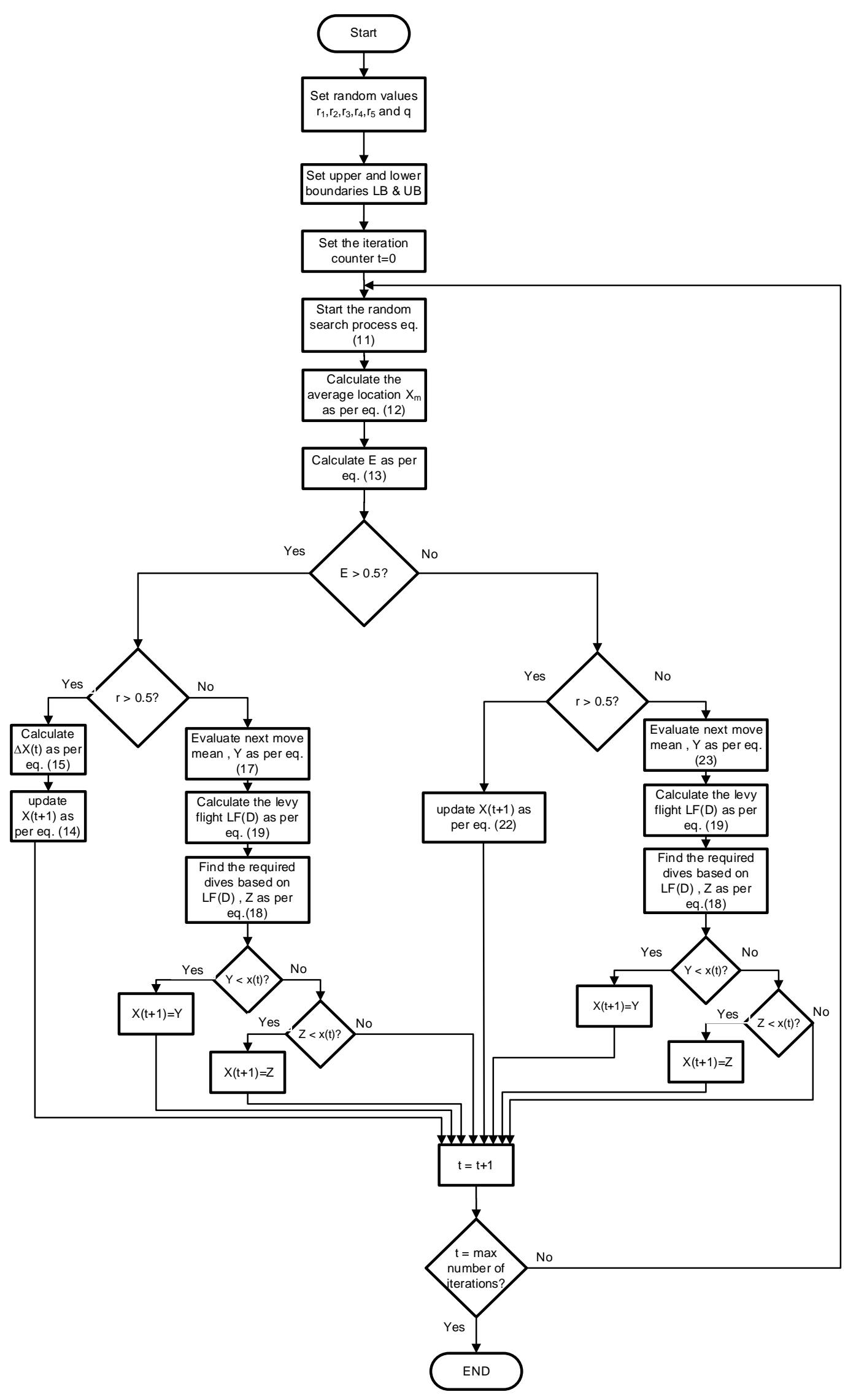

Figure 9. HHO algorithm flowchart. 


\section{Study Cases and Results Analysis}

The IEEE 30 bus test system shown in Figure 10 is used for evaluating the proposed OPF algorithms. This system consists of six generating units, four transformers with tap changing units, and nine reactive power compensation units-in addition to 41 transmission lines. The total active power demand of the network is $283.4 \mathrm{MW}$, while the total reactive power demand is 126.2 MVAR. Figure 10 shows the single line diagram of the IEEE 30 bus system, while Tables $2-4$ show the data of its components.

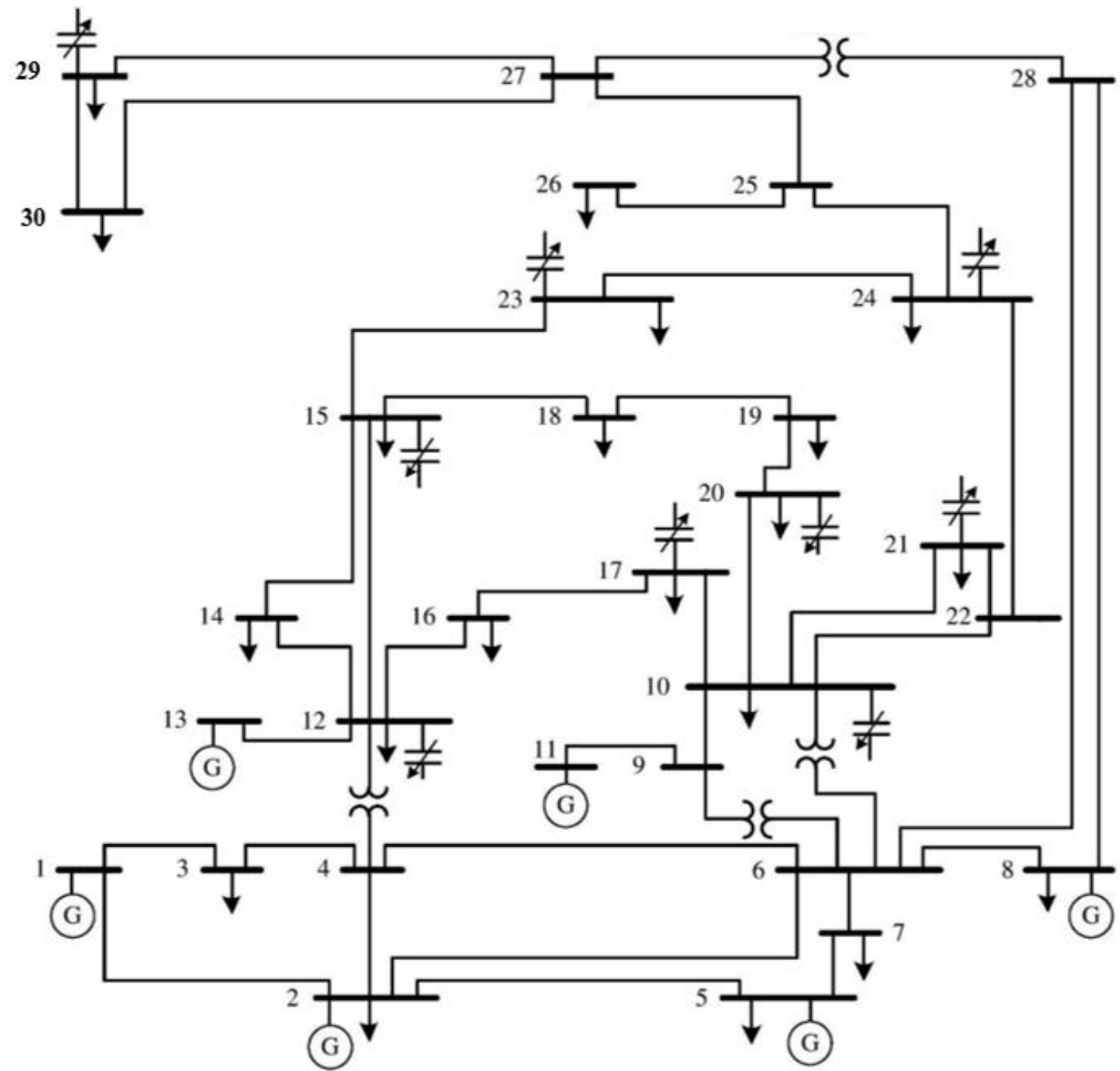

Figure 10. Single line diagram of the IEEE 30 bus system. 
In this section, four case studies were presented to assess the performance during different operating modes. In the first case, the objective of the OPF was to reduce the cost, while the second cases show the performance of the OPF algorithm when the objective was minimizing the transmission losses. Case 3 presents the behavior of the OPF proposed algorithm when it aims to reduce the voltage drop of transmission lines. The last case simulates the action of the OPF proposed algorithm when it has three objectives that minimize the cost, power losses, and voltage drops. The performance of the three proposed algorithms is compared with the particle swarm optimization (PSO) algorithm since it is a famous technique in solving the OPF problem. All simulations are performed using MATLAB.

Table 2. Generator data of the IEEE 30 bus system.

\begin{tabular}{cccccccc}
\hline \multirow{2}{*}{ Bus Tag } & \multicolumn{2}{c}{ Active Power Limits } & \multicolumn{2}{c}{ Reactive Power Limits } & \multicolumn{2}{c}{ Cost Factors } \\
\cline { 2 - 7 } & $\begin{array}{c}\text { Max. Active } \\
\text { Power (p.u.) }\end{array}$ & $\begin{array}{c}\text { Min. Active } \\
\text { Power (p.u.) }\end{array}$ & $\begin{array}{c}\text { Max. Reactive } \\
\text { Power (p.u.) }\end{array}$ & $\begin{array}{c}\text { Min. Reactive } \\
\text { Power (p.u.) }\end{array}$ & $\boldsymbol{a}$ & $\boldsymbol{b}$ & $\boldsymbol{c}$ \\
\hline 1 & 0.5 & 2.5 & -0.2 & 2 & 1 & 0 & 200 \\
2 & 0.2 & 0.8 & -0.2 & 0.8 & 0 & 175 \\
5 & 0.15 & 0.5 & -0.15 & 0.6 & 0 & 100 & 625 \\
8 & 0.1 & 0.35 & -0.15 & 0.5 & 0 & 325 & 83.4 \\
11 & 0.1 & 0.3 & -0.1 & 0.6 & 0 & 300 & 250 \\
13 & 0.12 & 0.4 & -0.15 & & & 250 \\
\hline
\end{tabular}

Table 3. Load data of the IEEE 30 bus system.

\begin{tabular}{ccc}
\hline Bus Tag & Active Power (p.u.) & Reactive Power (p.u.) \\
\hline 1 & 0 & 0 \\
2 & 0.217 & 0.127 \\
3 & 0.024 & 0.012 \\
4 & 0.076 & 0.016 \\
5 & 0.942 & 0.190 \\
6 & 0 & 0 \\
7 & 0.228 & 0.109 \\
8 & 0.300 & 0.300 \\
9 & 0 & 0 \\
10 & 0.058 & 0.020 \\
11 & 0 & 0 \\
12 & 0.112 & 0.075 \\
13 & 0 & 0 \\
14 & 0.062 & 0.016 \\
15 & 0.082 & 0.025 \\
16 & 0.035 & 0.018 \\
17 & 0.090 & 0.058 \\
18 & 0.032 & 0.009 \\
19 & 0.095 & 0.034 \\
20 & 0.022 & 0.007 \\
21 & 0.175 & 0.112 \\
22 & 0 & 0 \\
23 & 0.032 & 0.016 \\
24 & 0.087 & 0.067 \\
25 & 0 & 0 \\
26 & 0.035 & 0.023 \\
27 & 0 & 0 \\
28 & 0 & 0 \\
29 & 0.024 & 0.009 \\
30 & 0.106 & 0.019 \\
\hline
\end{tabular}


Table 4. Transmission network data of the IEEE 30 bus system.

\begin{tabular}{|c|c|c|c|c|c|c|}
\hline Line Tag & from Bus & to Bus & R (p.u.) & $X$ (p.u.) & B (p.u.) & Tap Settings \\
\hline 1 & 1 & 2 & 0.0192 & 0.0575 & 0.0264 & \\
\hline 2 & 1 & 3 & 0.0452 & 0.1852 & 0.0204 & \\
\hline 3 & 2 & 4 & 0.0570 & 0.1737 & 0.0184 & \\
\hline 4 & 3 & 4 & 0.0132 & 0.0379 & 0.0042 & \\
\hline 5 & 2 & 5 & 0.0472 & 0.1983 & 0.0209 & \\
\hline 6 & 2 & 6 & 0.0581 & 0.1763 & 0.0187 & \\
\hline 7 & 4 & 6 & 0.0119 & 0.0414 & 0.0045 & \\
\hline 8 & 5 & 7 & 0.0460 & 0.1160 & 0.0102 & \\
\hline 9 & 6 & 7 & 0.0267 & 0.0820 & 0.0085 & \\
\hline 10 & 6 & 8 & 0.0120 & 0.0420 & 0.0045 & \\
\hline 11 & 6 & 9 & 0 & 0.2080 & 0 & 1.078 \\
\hline 12 & 6 & 10 & 0 & 0.5560 & 0 & 1.069 \\
\hline 13 & 9 & 11 & 0 & 0.2080 & 0 & \\
\hline 14 & 9 & 10 & 0 & 0.1100 & 0 & \\
\hline 15 & 4 & 12 & 0 & 0.2560 & 0 & 1.032 \\
\hline 16 & 12 & 13 & 0 & 0.1400 & 0 & \\
\hline 17 & 12 & 14 & 0.1231 & 0.2559 & 0 & \\
\hline 18 & 12 & 15 & 0.0662 & 0.1304 & 0 & \\
\hline 19 & 12 & 16 & 0.0945 & 0.1987 & 0 & \\
\hline 20 & 14 & 15 & 0.2210 & 0.1997 & 0 & \\
\hline 21 & 16 & 17 & 0.0824 & 0.1932 & 0 & \\
\hline 22 & 15 & 18 & 0.1070 & 0.2185 & 0 & \\
\hline 23 & 18 & 19 & 0.0639 & 0.1292 & 0 & \\
\hline 24 & 19 & 20 & 0.0340 & 0.0680 & 0 & \\
\hline 25 & 10 & 20 & 0.0936 & 0.2090 & 0 & \\
\hline 26 & 10 & 17 & 0.0324 & 0.0845 & 0 & \\
\hline 27 & 10 & 21 & 0.0348 & 0.0749 & 0 & \\
\hline 28 & 10 & 22 & 0.0727 & 0.1499 & 0 & \\
\hline 29 & 21 & 22 & 0.0116 & 0.0236 & 0 & \\
\hline 30 & 15 & 23 & 0.1000 & 0.2020 & 0 & \\
\hline 31 & 22 & 24 & 0.1150 & 0.1790 & 0 & \\
\hline 32 & 23 & 24 & 0.1320 & 0.2700 & 0 & \\
\hline 33 & 24 & 25 & 0.1885 & 0.3292 & 0 & \\
\hline 34 & 25 & 26 & 0.2544 & 0.3800 & 0 & \\
\hline 35 & 25 & 27 & 0.1093 & 0.2087 & 0 & \\
\hline 36 & 28 & 27 & 0 & 0.3960 & 0 & 1.068 \\
\hline 37 & 27 & 29 & 0.2198 & 0.4153 & 0 & \\
\hline 38 & 27 & 30 & 0.3202 & 0.6027 & 0 & \\
\hline 39 & 29 & 30 & 0.2399 & 0.4533 & 0 & \\
\hline 40 & 8 & 28 & 0.0636 & 0.2000 & 0.0214 & \\
\hline 41 & 6 & 28 & 0.0169 & 0.0599 & 0.0065 & \\
\hline
\end{tabular}

\subsection{Case 1: Reducing Operating Costs}

In this case, the proposed algorithms aim to reduce the operating cost by minimizing the consumption of generator fuel, which means that Equation (18) is selected as the objective function for the OPF algorithm. Table 5 shows the optimal results of the three proposed algorithms. All the obtained results are compared with those received from the PSO algorithms. The results shown in Table 5 clearly show the effectiveness of all proposed techniques compared with the PSO. The MVO algorithm has succeeded in obtaining lower costs than the PSO by $2.1 \%$, while the GOA has lowered costs by $2.3 \%$. The HHO algorithm gave the lowest cost, which was lower than the PSO by $2.9 \%$. As shown in Figure 11, the $\mathrm{HHO}$ algorithm has an outstanding performance in terms of convergence speed as it converged after only 12 iterations. 
Table 5. Case 1 results.

\begin{tabular}{ccccc}
\hline Parameter & PSO & MVO & GOA & HHO \\
\hline$P_{G 2}$ (MW) & 31.9013 & 51.189 & 48.0194 & 47.4072 \\
$P_{G 5}(\mathrm{MW})$ & 15 & 21.311 & 20.9145 & 17.3766 \\
$P_{G 8}$ (MW) & 10 & 21.173 & 20.2342 & 17.8064 \\
$P_{G 11}$ (MW) & 29.9591 & 22.699 & 15.726 & 13.178 \\
$P_{G 13}$ (MW) & 12 & 16.587 & 13.5828 & 17.1314 \\
$V_{G 1}$ (p.u.) & 1.0816 & 1.0813 & 1.09356 & 1.074098 \\
$V_{G 2}$ (p.u.) & 1.058 & 1.0689 & 1.040936 & 1.049792 \\
$V_{G 5}$ (p.u.) & 1.0243 & 1.0406 & 0.969193 & 1.034415 \\
$V_{G 8}$ (p.u.) & 1.0358 & 1.0442 & 0.987262 & 1.028879 \\
$V_{G 11}$ (p.u.) & 1.0904 & 1.0748 & 1.029317 & 1.073113 \\
$V_{G 13}$ (p.u.) & 1.0048 & 1.0111 & 1.001084 & 1.071942 \\
$T_{11}$ (p.u.) & 1.0755 & 1.0525 & 1.066983 & 1.00642 \\
$T_{12}$ (p.u.) & 1.0293 & 0.96021 & 1.084914 & 0.999966 \\
$T_{15}$ (p.u.) & 0.9397 & 0.94864 & 0.910429 & 1.092749 \\
$T_{36}$ (p.u.) & 0.937 & 0.98519 & 0.973125 & 1.029863 \\
$Q_{c 10}$ (MVAR) & 0 & 2.4893 & 02.2169 & 2.8104 \\
$Q_{c 12}$ (MVAR) & 0 & 1.3383 & 0.5252 & 1.9302 \\
$Q_{c 15}$ (MVAR) & 0.3219 & 1.8017 & 4.522 & 2.7361 \\
$Q_{c 17}$ (MVAR) & 2.1412 & 0.1313 & 0.3904 & 1.5989 \\
$Q_{c 20}$ (MVAR) & 5 & 3.345 & 2.5788 & 01.472 \\
$Q_{c 21}$ (MVAR) & 0 & 0.482 & 0.7132 & 0.6965 \\
$Q_{c 23}$ (MVAR) & 0.4034 & 0.9994 & 2.2812 & 1.1422 \\
$Q_{c 24}$ (MVAR) & 2.2741 & 3.2872 & 4.3131 & 1.0534 \\
$Q_{c 29}$ (MVAR) & 0 & 0.041053 & 1.1918 & 1.6582 \\
Cost (\$/h) $_{P_{\text {loss }} \text { (kW) }}^{\mathbf{8 2 8 . 1 3 1 5}}$ & $\mathbf{8 1 0 . 9 0 1 1}$ & $\mathbf{8 0 9 . 7 4 1}$ & $\mathbf{8 0 4 . 1 4 0 7}$ \\
Voltage deviation (p.u) & 8.35021 & 7.68 & 10.09 & 7.97 \\
\hline & 0.77411 & 0.3751 & 0.7165 & 0.3909 \\
\hline & & & &
\end{tabular}

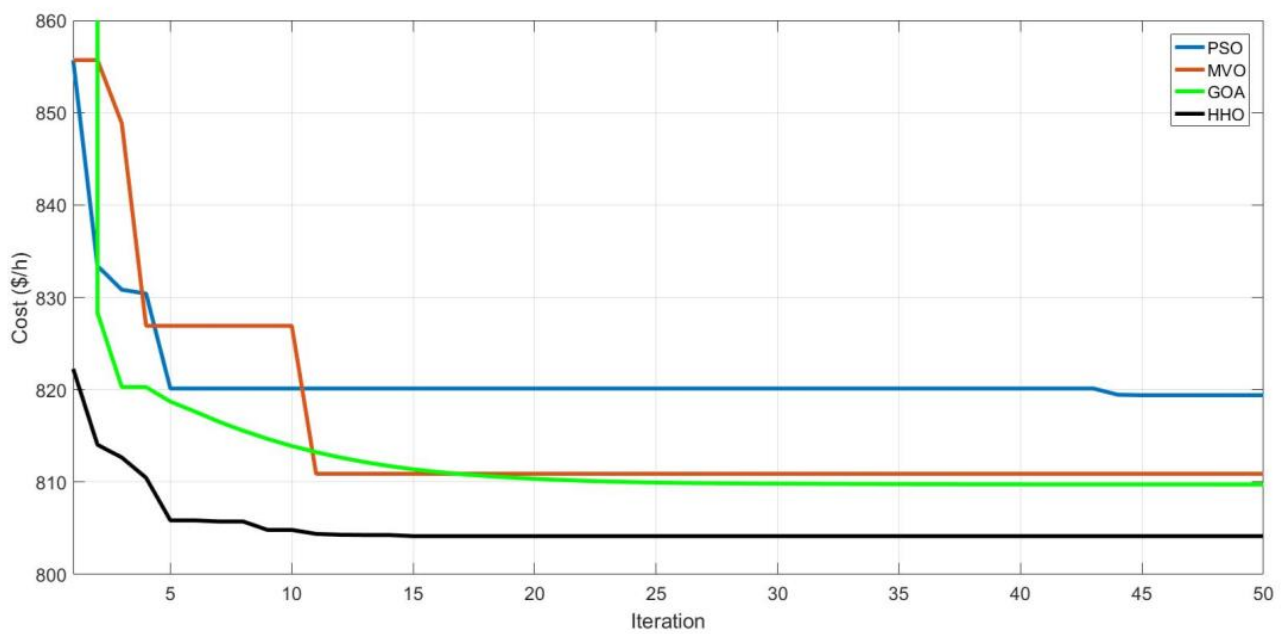

Figure 11. The convergence curve of the proposed algorithms in Case 1.

\subsection{Case 2: Reducing Power Losses}

The proposed algorithms are required to lower the power losses, which means that Equation (19) is selected as the objective function for the OPF algorithm. As shown in Table 6, all proposed algorithms have succeeded in reducing the power losses and gave better results than the PSO algorithm, which is usually used in the literature.

Both MVO and GOA have set the power system parameters to give lower losses than the PSO by $5.9 \%$ and $44.1 \%$, respectively. Again, the $\mathrm{HHO}$ succeeded in giving the best results, which are 56\% lower than the PSO. As indicated in Figure 12, the GOA was the fastest algorithm to reach the convergence, followed by the $\mathrm{PSO}$, the $\mathrm{HHO}$, and the MVO. As a general evaluation for the performance of the proposed algorithms in terms of 
accuracy and speed of convergence, the $\mathrm{HHO}$ is considered the best as it gave the lowest power losses after only 24 iterations.

Table 6. Case 2 results.

\begin{tabular}{ccccc}
\hline Parameter & PSO & MVO & GOA & HHO \\
\hline$P_{G 2}($ MW) & 80 & 25.857 & 68.6191 & 43.171 \\
$P_{G 5}$ (MW) & 50 & 25.789 & 50 & 50 \\
$P_{G 8}$ (MW) & 10 & 21.272 & 27.51 & 29.936 \\
$P_{G 11}$ (MW) & 10 & 28.111 & 17.0215 & 30 \\
$P_{G 13}$ (MW) & 40 & 12.963 & 24.6921 & 28.828 \\
$V_{G 1}$ (p.u.) & 1.1 & 1.0526 & 1.033698 & 1.0643 \\
$V_{G 2}$ (p.u.) & 1.0332 & 1.0339 & 1.005346 & 1.0571 \\
$V_{G 5}$ (p.u.) & 0.95 & 0.99197 & 0.958352 & 1.0291 \\
$V_{G 8}$ (p.u.) & 0.95 & 1.0074 & 0.998216 & 1.0187 \\
$V_{G 11}$ (p.u.) & 1.0760 & 0.9786 & 1.01605 & 1.1 \\
$V_{G 13}$ (p.u.) & 1.0403 & 1.0002 & 1.026657 & 1.0776 \\
$T_{11}$ (p.u.) & 0.9 & 0.96589 & 0.94774 & 1.0997 \\
$T_{12}$ (p.u.) & 1.1 & 1.0149 & 1.099986 & 0.94546 \\
$T_{15}$ (p.u.) & 0.9 & 1.0124 & 1.091799 & 1.0613 \\
$T_{36}$ (p.u.) & 0.9 & 0.90983 & 0.935101 & 0.96312 \\
$Q_{c 10}$ (MVAR) & 0 & 0.584 & 0.04772 & 0.047535 \\
$Q_{c 12}$ (MVAR) & 5 & 1.477 & 0.003591 & 0.05 \\
$Q_{c 15}$ (MVAR) & 5 & 1.5846 & 0.024723 & 0.05 \\
$Q_{c 17}$ (MVAR) & 0 & 2.4514 & 0.043997 & 0.011847 \\
$Q_{c 20}$ (MVAR) & 0.6688 & 2.2816 & 0.035 & 0.047438 \\
$Q_{c 21}$ (MVAR) & 2.6111 & 2.1068 & 0.041703 & 0.019112 \\
$Q_{c 23}$ (MVAR) & 5 & 4.5074 & 0.034738 & 0.023882 \\
$Q_{c 24}$ (MVAR) & 0 & 4.6145 & 0.044108 & 0.05 \\
$Q_{c 29}$ (MVAR) & 0 & 2.2979 & 0.027013 & 0.024811 \\
$\boldsymbol{P}_{\text {loss }}$ (MW) & $\mathbf{1 0 . 3 8 2 2}$ & $\mathbf{9 . 7 7}$ & $\mathbf{5 . 8 1}$ & $\mathbf{4 . 5 6}$ \\
Cost (\$/h) $_{\text {Moltage deviation (p.u) }}$ & 931.22 & 817.1171 & 878.8137 & 915.0934 \\
\hline & 0.4707 & 0.3545 & 0.3806 & 0.4794 \\
\hline
\end{tabular}

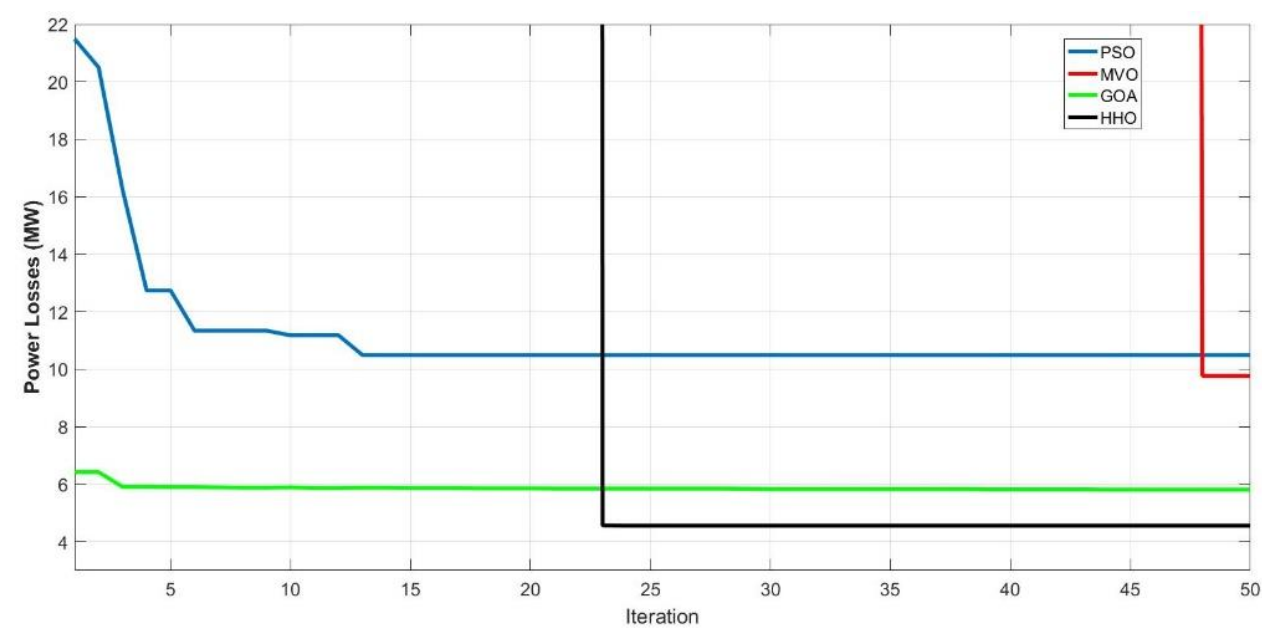

Figure 12. The convergence curve of the proposed algorithms in Case 2.

\subsection{Case 3: Reducing Voltage Drops}

In this case, the proposed algorithms aim to reduce the voltage drops at each bus. This means that Equation (20) is selected as the objective function for the OPF algorithm. Table 7 shows the optimal results of the three proposed algorithms. All the obtained results are compared with those received from the PSO algorithms. The results shown in Table 7 clearly show the effectiveness of all proposed techniques compared with the PSO. The MVO algorithm has succeeded in obtaining costs lower than the PSO by $30.2 \%$, while the 
GOA has lowered the cost by $51.3 \%$. The HHO algorithm gave the lowest cost, which was lower than the PSO by 51\%. As shown in Figure 13, the HHO algorithm has an outstanding performance in terms of convergence speed as it converged after only one iteration. It is essential to mention that the $\mathrm{HHO}$ algorithm has been tested many times, and at each attempt, it gave the same response.

Table 7. Case 3 results.

\begin{tabular}{ccccc}
\hline Parameter & PSO & MVO & GOA & HHO \\
\hline$P_{G 2}$ (MW) & 80 & 22.258 & 55.8604 & 56.607 \\
$P_{G 5}$ (MW) & 15 & 50 & 15 & 23.951 \\
$P_{G 8}$ (MW) & 17.7333 & 28.73 & 17.6747 & 14.786 \\
$P_{G 11}(\mathrm{MW})$ & 10 & 24.666 & 24.3688 & 17.467 \\
$P_{G 13}$ (MW) & 16.0582 & 14.501 & 24.7847 & 19.229 \\
$V_{G 1}$ (p.u.) & 1.0696 & 1.0603 & 1.070652 & 1.0259 \\
$V_{G 2}$ (p.u.) & 1.0499 & 1.0535 & 1.041199 & 1.0157 \\
$V_{G 5}$ (p.u.) & 1.0346 & 1.0104 & 0.992444 & 1.0076 \\
$V_{G 8}$ (p.u.) & 1.0017 & 0.99797 & 1.025342 & 1.0107 \\
$V_{G 11}$ (p.u.) & 0.95 & 0.99467 & 1.008014 & 1.0007 \\
$V_{G 13}$ (p.u.) & 1.1 & 1.0872 & 1.034387 & 1.0068 \\
$T_{11}$ (p.u.) & 1.038 & 1.0193 & 1.00306 & 0.97984 \\
$T_{12}$ (p.u.) & 1.0709 & 1.0604 & 0.958199 & 0.96271 \\
$T_{15}$ (p.u.) & 1.0367 & 1.0182 & 1.012606 & 1.0038 \\
$T_{36}$ (p.u.) & 0.9577 & 0.93723 & 0.988767 & 0.96339 \\
$Q_{c 10}$ (MVAR) & 5 & 02.7467 & 4.742 & 4.9891 \\
$Q_{c 12}$ (MVAR) & 3.0157 & 0.3608 & 3.956 & 4.6581 \\
$Q_{c 15}$ (MVAR) & 0 & 3.0522 & 4.5351 & 4.989 \\
$Q_{c 17}$ (MVAR) & 0 & 0.4881 & 3.73 & 4.9891 \\
$Q_{c 20}$ (MVAR) & 0 & 2.2206 & 1.918 & 3.4195 \\
$Q_{c 21}$ (MVAR) & 5 & 3.1629 & 2.9936 & 4.9891 \\
$Q_{c 23}$ (MVAR) & 0.8774 & 0.4416 & 4.5447 & 4.9891 \\
$Q_{c 24}$ (MVAR) & 5 & 1.8394 & 3.7481 & 3.4275 \\
$Q_{c 29}$ (MVAR) & 0 & 2.2979 & 4.1399 & 2.9353 \\
Voltage deviation (p.u) & $\mathbf{0 . 5 1 7 8}$ & $\mathbf{0 . 3 6 1 2}$ & $\mathbf{0 . 2 5 2 1}$ & $\mathbf{0 . 1 4 9 4}$ \\
Cost (EGP/h) & 827.93 & 862.7863 & 825.7566 & 849.8061 \\
$P_{\text {loss }}$ (MW) & 10.19937 & 9.04 & 9.77 & 5.79 \\
\hline
\end{tabular}

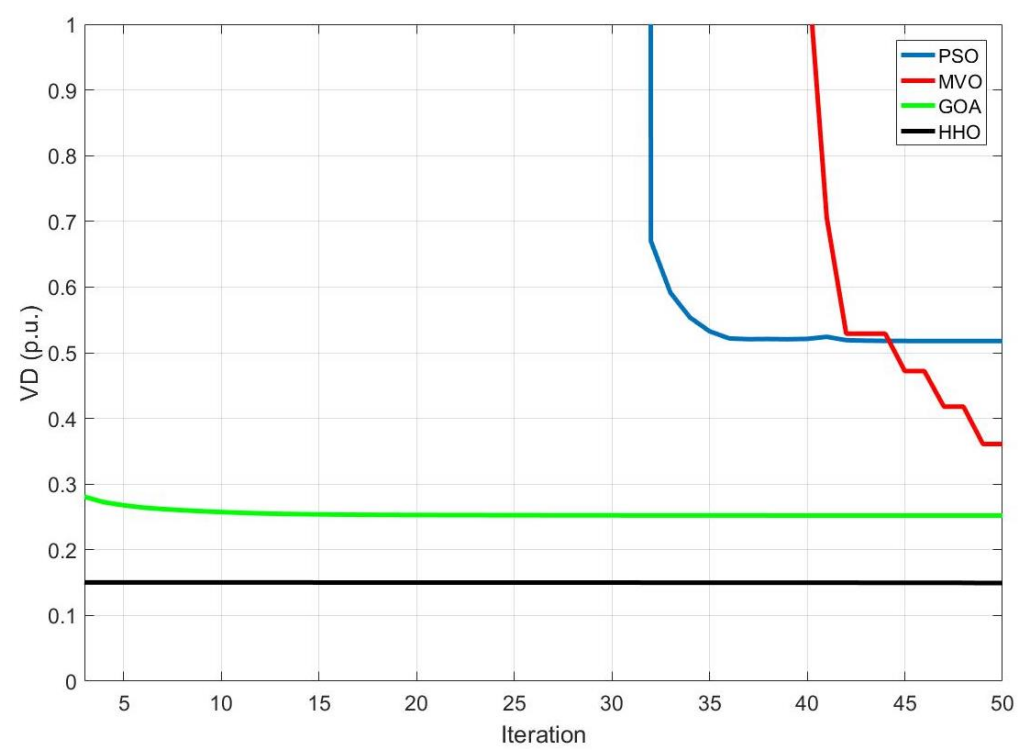

Figure 13. The convergence curve of the proposed algorithms in Case 3. 
4.4. Case 4: Reducing the Operation Costs, the Power Losses, and the Transmission Voltage Drops

In previous cases, the proposed algorithms have worked to optimize a single objective: either the operating costs or the power losses, or even the voltage drops. However, this has affected the other parameters. For example, in Table 5, the GOA algorithm succeeded in lowering the cost. However, based on these parameters, the voltage will drop to reach 0.71 p.u. - which is not acceptable. To solve this problem, the proposed algorithms in Case 4 use Equation (21) as a multi-objective function which is utilized to update the parameters to lower the operation costs, the power losses, and the voltage drops. As indicated in Table 8, the proposed algorithms have given better results compared to the PSO.

Table 8. Case 4 results.

\begin{tabular}{ccccc}
\hline Parameter & PSO & MVO & GOA & HHO \\
\hline$P_{G 2}$ (MW) & 77.4361 & 23.413 & 49.9138 & 32.061 \\
$P_{G 5}$ (MW) & 50 & 16.143 & 36.4247 & 32.2866 \\
$P_{G 8}$ (MW) & 35 & 10.81 & 28.86 & 31.7724 \\
$P_{G 11}(\mathrm{MW})$ & 30 & 27.172 & 22.4329 & 27.3033 \\
$P_{G 13}$ (MW) & 33.7972 & 28.851 & 27.6338 & 22.9216 \\
$V_{G 1}$ (p.u.) & 0.95 & 1.0647 & 1.058299 & 1.048423 \\
$V_{G 2}$ (p.u.) & 0.95 & 1.0393 & 1.018794 & 1.038553 \\
$V_{G 5}$ (p.u.) & 0.95 & 0.99051 & 0.966312 & 1.004721 \\
$V_{G 8}$ (p.u.) & 0.95 & 1.0047 & 1.00055 & 1.00622 \\
$V_{G 11}$ (p.u.) & 1.0659 & 1.0791 & 1.027062 & 1.048021 \\
$V_{G 13}$ (p.u.) & 1.1 & 1.035 & 0.984915 & 1.014238 \\
$T_{11}$ (p.u.) & 1.1 & 1.0575 & 0.923636 & 0.966222 \\
$T_{12}$ (p.u.) & 1.1 & 1.0613 & 0.990273 & 1.017401 \\
$T_{15}$ (p.u.) & 0.9 & 0.94474 & 1.003333 & 0.945139 \\
$T_{36}$ (p.u.) & 0.9 & 0.95878 & 0.959267 & 0.981819 \\
$Q_{c 10}$ (MVAR) & 5 & 2.2919 & 4.2931 & 1.3612 \\
$Q_{c 12}$ (MVAR) & 0 & 3.4055 & 0.0594 & 1.2633 \\
$Q_{c 15}$ (MVAR) & 5 & 4.8883 & 3.4643 & 2.9683 \\
$Q_{c 17}$ (MVAR) & 5 & 1.1817 & 0.3222 & 1.474 \\
$Q_{c 20}$ (MVAR) & 5 & 0.7682 & 2.4384 & 4.2129 \\
$Q_{c 21}$ (MVAR) & 5 & 4.1172 & 4.406 & 2.0813 \\
$Q_{c 23}$ (MVAR) & 5 & 2.1211 & 3.8883 & 2.174 \\
$Q_{c 24}$ (MVAR) & 0 & 2.8667 & 4.2725 & 2.0423 \\
$Q_{c 29}$ (MVAR) & 5 & 2.4113 & 1.9045 & 1.7752 \\
Cost (EGP/h) $_{P_{l o s s} \text { (MW) }}$ & 952.56 & 831.5875 & 841.3947 & 830.8591 \\
Voltage deviation (p.u) & 5.2514 & 10.04 & 6.83 & 6.75 \\
\hline & 0.62144 & 0.285 & 0.3421 & 0.2438 \\
\hline
\end{tabular}

This case clearly shows the limitations of each method of the proposed methods applied to a multi-objective optimization function. Although the performance of the MVO was satisfactory in previous cases (as shown in Figure 14), it failed to reach the global optimum solution. Instead, it reached the optimum local value. Additionally, in terms of speed of conversion, it took too many iterations to reach convergence. Regarding the GOA, it also does not reach the best solutions for the three objectives. However, the speed of convergence is much better than the MVO. The HHO is the only algorithm that best performs as it finds global optimum solutions for the three objectives after only five iterations. 


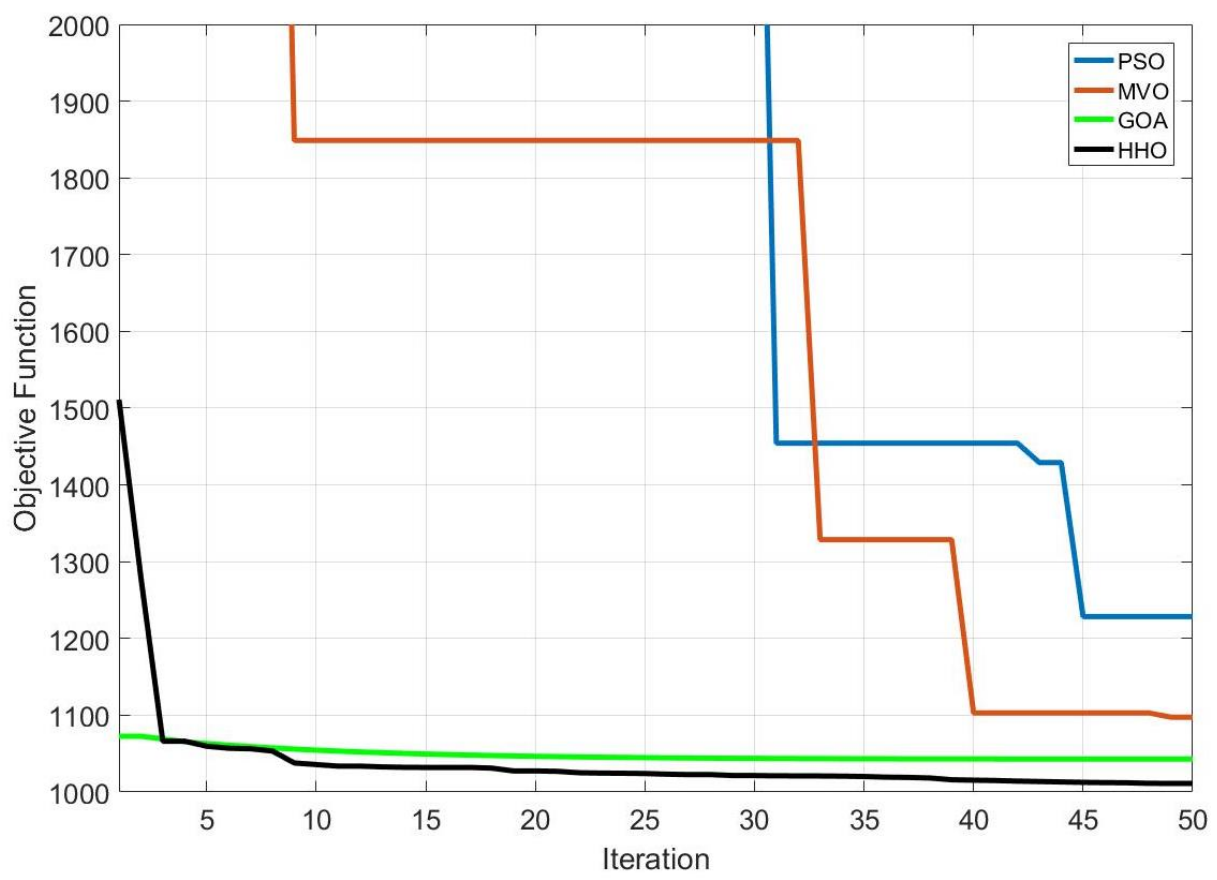

Figure 14. The convergence curve of the proposed algorithms in Case 4.

\section{Conclusions}

In this paper, three naturally inspired meta-heuristic algorithms were proposed to solve the OPF problem. Detailed mathematical modeling for the OPF problem was presented, including the power flow's multi-objective optimization by reducing operation fuel costs, transmission power losses, and voltage drops. The proposed techniques were simulated using MATLAB and applied to the IEEE 30 bus bench-mark system to show the effectiveness of each algorithm. Four case studies were formulated to assess the performance of each algorithm. In each case, the results of the proposed algorithms were compared with the PSO algorithm, which is commonly used in the literature to solve the OPF problem. The HHO algorithm showed the best performance in achieving a minimum cost in the first scenario, where it saved USD 24/h compared to the PSO. However, in the second and third scenarios, the proposed $\mathrm{HHO}$ algorithm successfully resulted in $6 \mathrm{MW}$ less power loss and 0.37 p.u. more minor voltage deviation when compared to PSO results. Finally, even in the multi-objective scenario, the proposed $\mathrm{HHO}$ proved to be a reliable algorithm compared to all other algorithms under investigation. Research findings show that the HHO algorithm may form a very competitive algorithm for power system optimization problems.

Author Contributions: M.A. contributed to the research framework, checked, and revised draft paper; H.D. collected the data and wrote the draft manuscript, checked, and revised the paper; M.A. supervised the whole process. A.A. ead and approved the final manuscript. All authors have read and agreed to the published version of the manuscript.

Funding: This research received no external funding.

Institutional Review Board Statement: Not applicable.

Informed Consent Statement: Not applicable.

Data Availability Statement: No new data were created or analyzed in this study. Data sharing is not applicable to this article.

Conflicts of Interest: The authors declare no conflict of interest. 


\section{References}

1. Carpentier, J. Contribution to the economic dispatch problem. Bull. Soc. Fr. Electr. 1962, 8, 431-447.

2. Dommel, H.; Tinney, W. Optimal power flow solutions. IEEE Trans. Power Appar. Syst. 1968, 87, 1866-1876. [CrossRef]

3. Frank, S.; Steponavice, I.; Rebennack, S. Optimal power flow: A bibliographic survey II Non-deterministic and hybrid methods. Energy Syst. 2012, 3, 259-289. [CrossRef]

4. Niu, M.; Wan, C.; Xu, Z. A review on applications of heuristic optimization algorithms for optimal power flow in modern power systems. J. Mod. Power Syst. Clean Energy 2014, 2, 289-297. [CrossRef]

5. Abou El Ela, A.A.; Abido, M.A.; Spea, S.R. Optimal power flow using dif-ferential evolution algorithm. Electr. Eng. 2009, 91, 69-78. [CrossRef]

6. Alsac, O.; Stott, B. Optimal load flow with steady-state security. IEEE Trans. Power Appar. Syst. 1974. [CrossRef]

7. Shoults, R.R.; Sun, D.T. Optimal power flow based upon P-Q decomposition. IEEE Trans. Power Appar. Syst. 1982. [CrossRef]

8. Sun, D.I.; Ashley, B.; Brewer, B.; Hughes, A.; Tinney, W.F. Optimal power flow by Newton approach. IEEE Trans. Power Appar. Syst. 1984, PAS-103, 2864-2880. [CrossRef]

9. Burchett, R.C.; Happ, H.H.; Vierath, D.R. Quadratically Convergent Optimal Power Flow. IEEE Trans. Power Appar. Syst. 1984. [CrossRef]

10. Abido, M.A. Optimal power flow using particle swarm optimization. Electr. Power Energy Syst. 2002, 24, 563-571. [CrossRef]

11. He, S.; Wen, J.Y.; Prempain, E.; Wu, Q.H.; Fitch, J.; Mann, S. An improved particle swarm optimization for optimal power flow. In Proceedings of the 2004 International Conference on Power System Technology, Singapore, 21-24 November 2004; pp. 1633-1637.

12. Zhao, B.; Guo, C.; Cao, Y. Improved particle swam optimization algorithm for OPF problems. In Proceedings of the IEEE PES Power Systems Conference and Exposition, New York, NY, USA, 10-13 October 2004; pp. 233-238.

13. Wang, C.R.; Yuan, H.J.; Huang, Z.Q.; Zhang, J.W.; Sun, C.J. A modified particle swarm optimization algorithm and its application in optimal power flow pro-blem. In Proceedings of the 4th International Conference on Machine Learning and Cybernetics, Guangzhou, China, 18-21 August 2005; pp. 2885-2889.

14. Lai, L.L.; Ma, J.T.; Yokoyama, R.; Zhao, M. Improved genetic algorithms for optimal power flow under both normal and contingent operation States. Int. J. Electr. Power Energy Syst. 1997. [CrossRef]

15. Bakirtzis, A.G.; Biskas, P.N.; Zoumas, C.E.; Petridis, V. Optimal power flow by enhanced genetic algorithm. IEEE Trans. Power Syst. 2002. [CrossRef]

16. Kumari, M.S.; Maheswarapu, S. Enhanced Genetic Algorithm based computation technique for multi-objective Optimal Power Flow solution. Int. J. Electr. Power Energy Syst. 2010. [CrossRef]

17. Niknam, T.; Narimani, M.R.; Aghaei, J.; Azizipanah-Abarghooee, R. Improved particle swarm optimisation for multi-objective optimal power flow considering the cost, loss, emission and voltage stability index. IET Gener. Transm. Distrib. 2012. [CrossRef]

18. Bhattacharya, A.; Chattopadhyay, P.K. Application of biogeography-based optimisation to solve different optimal power flow problems. IET Gener. Transm. Distrib. 2011. [CrossRef]

19. Ghanizadeh, A.J.; Mokhtari, G.; Abedi, M.; Gharehpetian, G.B. Optimal power flow based on imperialist com-petitive algorithm. Int. Rev. Electr. Eng. 2011, 6, 1847-1852.

20. El-Fergany, A.A.; Hasanien, H.M. Single and Multi-objective Optimal Power Flow Using Grey Wolf Opti-mizer and Differential Evolution Algorithms. Electr. Power Compon. Syst. 2015. [CrossRef]

21. Radosavljević, J.; Klimenta, D.; Jevtić, M.; Arsić, N. Optimal Power Flow Using a Hybrid Optimization Algo-rithm of Particle Swarm Optimization and Gravitational Search Algorithm. Electr. Power Compon. Syst. 2015. [CrossRef]

22. Duman, S.; Güvenc, U.; Sönmez, Y.; Yörükeren, N. Optimal power flow using gravitational search algorithm. Energy Convers. Manag. 2012, 59, 86-95. [CrossRef]

23. Bhattacharya, A.; Roy, P.K. Solution of multi-objective optimal power flow using gravitational search algo-rithm. IET Gener. Transm. Distrib. 2012. [CrossRef]

24. Jahan, M.S.; Amjady, N. Solution of large-scale security constrained optimal power flow by a new bi-level optimisation approach based on enhanced gravitational search algorithm. IET Gener. Transm. Distrib. 2013. [CrossRef]

25. Bouchekara, H.R.E.H.; Abido, M.A. Optimal power flow using differential search algorithm. Electr. Power Compon. Syst. 2014. [CrossRef]

26. El-Sehiemy, R.A.; Shafiq, M.B.; Azmy, A.M. Multi-phase search optimisation algorithm for constrained opti-mal power flow problem. Int. J. Bio-Inspired Comput. 2014, 6, 275-289. [CrossRef]

27. Pulluri, H.; Naresh, R.; Sharma, V. A solution network based on stud krill herd algorithm for optimal power flow problems. Soft Comput. 2018. [CrossRef]

28. Liang, R.H.; Tsai, S.R.; Chen, Y.T.; Tseng, W.T. Optimal power flow by a fuzzy based hybrid particle swarm opti-mization approach. Electr. Power Syst. Res. 2011. [CrossRef]

29. Arul, R.; Ravi, G.; Velusami, S. Solving optimal power flow problems using chaotic self-adaptive differential harmony search algorithm. Electr. Power Compon. Syst. 2013. [CrossRef]

30. Bouchekara, H.R.E.H. Optimal power flow using black-hole-based optimization approach. Appl. Soft Comput. J. 2014. [CrossRef]

31. Sivasubramani, S.; Swarup, K.S. Multi-objective harmony search algorithm for optimal power flow prob-lem. Int. J. Electr. Power Energy Syst. 2011. [CrossRef]

32. Warid, W.; Hizam, H.; Mariun, N.; Abdul-Wahab, N.I. Optimal power flow using the Jaya algorithm. Energies 2016, 678. [CrossRef] 
33. Bouchekara, H.R.E.H.; Abido, M.A.; Boucherma, M. Optimal power flow using Teaching-Learning-Based Optimization technique. Electr. Power Syst. Res. 2014. [CrossRef]

34. Christy, A.A.; Raj, P.A.D.V. Adaptive biogeography based predator-prey optimization technique for optimal power flow. Int. J. Electr. Power Energy Syst. 2014. [CrossRef]

35. He, X.; Wang, W.; Jiang, J.; Xu, L. An improved artificial bee colony algorithm and its application to multi-objective optimal power flow. Energies 2015, 2412. [CrossRef]

36. Sanseverino, E.R.; Di Silvestre, M.L.; Badalamenti, R.; Nguyen, N.Q.; Guerrero, J.M.; Meng, L. Optimal power flow in islanded microgrids using a simple distributed algorithm. Energies 2015, 8, 11493-11514. [CrossRef]

37. Balachennaiah, P.; Suryakalavathi, M.; Nagendra, P. Firefly algorithm based solution to minimize the real power loss in a power system. Ain Shams Eng. J. 2018. [CrossRef]

38. Abdelsalam, M.; Diab, H.Y. Optimal Coordination of DOC Relays Incorporated into a Distributed Generation-Based Micro-Grid Using a Meta-Heuristic MVO Algorithm. Energies 2019, 12, 4115. [CrossRef]

39. Mirjalili, S.; Mirjalili, S.M.; Hatamlou, A. Multi-Verse Optimizer: A nature-inspired algorithm for global optimization. Neural Comput. Appl. 2016, 27, 495-513. [CrossRef]

40. Gad, Y.; Diab, H.; Abdelsalam, M.; Galal, Y. Smart Energy Management System of Environmentally Friendly Microgrid Based on Grasshopper Optimization Technique. Energies 2020, 13, 5000. [CrossRef]

41. Mafarja, M.; Aljarah, I.; Heidari, A.A.; Hammouri, A.I.; Faris, H.; Ala'M, A.-Z.; Mirjalili, S. Evolutionary population dynamics and grasshopper optimization approaches for feature selection problems. Knowl. Based Syst. 2018, 145, 25-45. [CrossRef]

42. Heidari, A.A.; Mirjalili, S.; Faris, H.; Aljarah, I.; Mafarja, M.; Chen, H. Harris hawks optimization: Algorithm and applications. Future Gener. Comput. Syst. 2019, 97, 849-872. [CrossRef] 